\title{
THE
}

\section{Impact of Breaking Wave Form Drag on Near-Surface Turbulence and Drag Coefficient over Young Seas at High Winds}

\author{
Nobuhiro Suzuki \\ University of Rhode Island \\ Tetsu Hara \\ University of Rhode Island, thara@uri.edu \\ Peter P. Sullivan
}

Follow this and additional works at: https://digitalcommons.uri.edu/gsofacpubs

\section{Citation/Publisher Attribution}

Suzuki, N., Hara, T., \& Sullivan, P. P. (2013). Impact of Breaking Wave Form Drag on Near-Surface Turbulence and Drag Coefficient over Young Seas at High Winds. J. Phys. Oceanogr., 43, 324-343. doi: 10.1175/JPO-D-12-0127.1

Available at: https://doi.org/10.1175/JPO-D-12-0127.1

This Article is brought to you for free and open access by the Graduate School of Oceanography at DigitalCommons@URI. It has been accepted for inclusion in Graduate School of Oceanography Faculty Publications by an authorized administrator of DigitalCommons@URI. For more information, please contact digitalcommons-group@uri.edu. 


\title{
Impact of Breaking Wave Form Drag on Near-Surface Turbulence and Drag Coefficient over Young Seas at High Winds
}

\author{
NobUhiro SuzUKi AND TETSU HARA \\ Graduate School of Oceanography, University of Rhode Island, Narragansett, Rhode Island \\ Peter P. Sullivan \\ National Center for Atmospheric Research, Boulder, Colorado
}

(Manuscript received 20 July 2012, in final form 23 October 2012)

\begin{abstract}
The effects of breaking waves on near-surface wind turbulence and drag coefficient are investigated using large-eddy simulation. The impact of intermittent and transient wave breaking events (over a range of scales) is modeled as localized form drag, which generates airflow separation bubbles downstream. The simulations are performed for very young sea conditions under high winds, comparable to previous laboratory experiments in hurricane-strength winds. The results for the drag coefficient in high winds range between about 0.002 and 0.003 . In such conditions more than $90 \%$ of the total air-sea momentum flux is due to the form drag of breakers; that is, the contributions of the nonbreaking wave form drag and the surface viscous stress are small. Detailed analysis shows that the breaker form drag impedes the shear production of the turbulent kinetic energy (TKE) near the surface and, instead, produces a large amount of small-scale wake turbulence by transferring energy from large-scale motions (such as mean wind and gusts). This process shortcuts the inertial energy cascade and results in large TKE dissipation (integrated over the surface layer) normalized by friction velocity cubed. Consequently, the large production of wake turbulence by breakers in high winds results in the small drag coefficient obtained in this study. The results also suggest that common parameterizations for the mean wind profile and the TKE dissipation inside the wave boundary layer, used in previous Reynolds-averaged Navier-Stokes models, may not be valid.
\end{abstract}

\section{Introduction}

In this paper, we investigate the turbulence in the atmospheric surface layer that develops over a field of breaking surface waves in hurricane-strength winds $\left(30-70 \mathrm{~m} \mathrm{~s}^{-1}\right)$. Such turbulence is important as it affects air-sea exchanges of momentum and heat as well as suspension and dispersion of sea-spray droplets and other passive tracers. These surface-layer processes are critical factors affecting larger-scale phenomena such as tropical cyclones. Despite their importance, the surfacelayer processes at high winds remain poorly understood due to the extreme air-sea conditions involved.

An outstanding question is how the drag coefficient $C_{D 10} \equiv\left(U_{*} / U_{10}\right)^{2}$ over the ocean depends on the wind

Corresponding author address: Nobuhiro Suzuki, Graduate School of Oceanography, University of Rhode Island, 215 South Ferry Rd., Narragansett, RI 02882.

E-mail: nobuhiro_suzuki@my.uri.edu speed at high wind speeds. Here, $U_{*}$ is the friction velocity, and $U_{10}$ is the mean wind speed at $10-\mathrm{m}$ height. In low to moderate winds, the drag coefficient is known to increase with the wind speed (e.g., Edson et al. 2007). However, in hurricane-strength winds, field observations suggest that the drag coefficient may saturate (i.e., stop increasing) or even decrease with the wind speed (Powell et al. 2003; French et al. 2007; Bell et al. 2012) and is much less than the extrapolations of the bulk relationships derived from the low to moderate wind observations. Similar dependence of the drag coefficient on the wind speed has been observed in a fixed-fetch windwave tank experiment (Donelan et al. 2004) as well.

The cause of the drag coefficient reduction remains unclear. Possible causes considered in the literature include sea foam, sea spray, and breaking waves. Sea foam (or foam spray) may affect the drag coefficient via altering the velocity boundary conditions for the surface layer (Powell et al. 2003; Soloviev and Lukas 2010; Holthuijsen et al. 2012). Sea spray is a potential cause 
because 1) its mass and its exchange of heat with surrounding air influence the stratification of the surface layer (e.g., Bianco et al. 2011; Bao et al. 2011; Kudryavtsev and Makin 2011), and 2) suspension of spray droplets results in turbulent kinetic energy (TKE) loss and effectively enhances the TKE dissipation rate (Makin 2005; Barenblatt et al. 2005). Both stratification and TKE dissipation rate may modify the turbulence affecting the drag coefficient. Lastly, breaking waves may play a role in the drag coefficient reduction because they affect the atmospheric wave boundary layer (WBL) dynamics. Here, the atmospheric WBL refers to the lower part of the surface layer where airflow is directly affected by waves. Previous theoretical studies of the WBL in high winds (Kudryavtsev and Makin 2007; Kukulka et al. 2007; Kukulka and Hara 2008b; Mueller and Veron 2009) investigated the "sheltering effect" due to airflow separation over breaking waves. Here, the sheltering effect refers to reduction of the viscous surface stress and the form drag of small roughness elements inside an airflow separation bubble formed by a larger breaking wave.

In addition to the above mechanisms, the drag coefficient may be reduced by the vigorous production of wake turbulence over breaking waves and the resultant shortcut of the energy cascade. In high winds, breakers may cause vigorous wake eddies (such as separation bubbles) whose sizes roughly scale with the breaker heights (e.g., Reul et al. 2008). Such wake production transfers energy from large-scale motions (viz., the mean wind and large-scale eddies) to small-scale turbulence near the viscous dissipation scale (i.e., it shortcuts the inertial energy cascade) and results in enhanced TKE dissipation (e.g., Shaw and Schumann 1992; Finnigan 2000). Although the importance of wakegenerated turbulence has been long recognized in studies of canopy-layer flows (e.g., Raupach and Shaw 1982), it has been overlooked in the previous theoretical studies of the WBL in high winds.

Another weakness of the previous theoretical WBL studies is that they are based on a Reynolds-averaged Navier-Stokes (RANS) modeling framework and use parameterizations that are originally developed for turbulence over flat walls. Some models (Kudryavtsev and Makin 2007; Mueller and Veron 2009) assume that the wind profile in the WBL is similar to the wind profiles over flat walls (viz., logarithmic or linear-logarithmic), and other models (Kukulka et al. 2007; Kukulka and Hara 2008b) assume that the transport and viscous dissipation terms in the TKE budget behave similarly to those over flat walls. However, the wind profile, the TKE transport, and the TKE dissipation are generally influenced by roughness elements such as breakers and may differ from those over flat walls. In fact, such modification has been observed in many types of roughness sublayers [e.g., Ikeda and Durbin (2007) for $k$-type roughness such as bars mounted transversely to the mean wind, Finnigan (2000) for plant canopies, and Britter and Hanna (2003) for urban canopies]. Thus, application of the flat-wall parameterizations to the WBL may not be valid.

Therefore, in this paper, we address two important questions regarding the atmospheric WBL in high winds: 1) how does the production of the wake turbulence by breaking waves modify the TKE budget, the mean wind, and the drag coefficient and 2) are the existing turbulence parameterizations in the WBL RANS models valid. These questions are answered by using large-eddy simulation (LES) that explicitly simulates intermittent and transient form drag and wake turbulence due to individual breakers. The advantage of such LES over RANS approaches is that it does not heavily rely on turbulence parameterizations other than the subgridscale parameterization. In contrast, RANS models have to parameterize the effects of wake turbulence. As our focus is on the breaker form drag and wakes, we will not consider sea foam, sea spray, heat flux, and stratification.

\section{Methods}

\section{a. LES model of the WBL with breaker effects}

Our LES employs an approach successfully used in large-eddy simulations of canopy-layer flows (e.g., Shaw and Schumann 1992) and upper-ocean boundary layer flows (e.g., Sullivan et al. 2007). In such an approach the actual geometry and motion of roughness elements are not resolved, but their impact is modeled by applying local and instantaneous forces that would result from the roughness elements. The force applied in the computational domain interior represents the form drag over intermittent breakers or, more precisely, the momentum exchange between the breakers and their surrounding air via the pressure force induced by the breakers. The LES equations are otherwise standard.

Namely, the governing equations for filtered (or resolved) motions and subgrid-scale (SGS) kinetic energy are (Deardorff 1980; Moeng 1984; Sullivan et al. 1994, 2007)

$$
\begin{aligned}
\frac{\partial \bar{u}_{i}}{\partial t} & =-\bar{u}_{j} \frac{\partial \bar{u}_{i}}{\partial x_{j}}-\frac{\partial \bar{p}}{\partial x_{i}}-\frac{\partial R_{i j}}{\partial x_{j}}-\frac{\partial \bar{P}}{\partial x_{i}}+\sum_{m} \bar{A}_{i}^{m}, \\
\frac{\partial \bar{u}_{i}}{\partial x_{i}} & =0, \\
\frac{\partial e}{\partial t} & =-\bar{u}_{j} \frac{\partial e}{\partial x_{j}}-R_{i j} \frac{\partial \bar{u}_{i}}{\partial x_{j}}+T^{\mathrm{SGS}}-\epsilon+\sum_{m} W^{m} .
\end{aligned}
$$


Here, filtered variables are denoted by an overbar; $x_{1}, x_{2}, x_{3}$ (or equivalently $x, y, z$ ) are the streamwise, spanwise, and vertical coordinates, respectively; $\left(u_{1}, u_{2}, u_{3}\right)=$ $(u, v, w)$ are the velocity components; $p$ is the pressure divided by the uniform density; $\bar{P}=\bar{P}\left(x_{1}\right)$ is an external large-scale forcing used to drive the flow, and $-\partial \bar{P} / \partial x_{1}$ is constant in time, uniform in space, and positive; $R_{i j} \equiv$ $\overline{u_{i} u_{j}}-\bar{u}_{i} \bar{u}_{j}$ is the SGS stress; $e \equiv\left(\overline{u_{i} u_{i}}-\bar{u}_{i} \bar{u}_{i}\right) / 2$ is the SGS kinetic energy; $T^{\mathrm{SGS}}$ is the SGS transport; $\epsilon$ is the viscous dissipation; $\bar{A}_{i}^{m}$ and $W^{m}$ are the momentum input to the resolved motion and the work done to the SGS turbulence in a local discrete breaking wave event $m$, respectively. We adopt a flat bottom idealization; that is, we employ a surface-fitted coordinate (Fig. 1a), but the equations are approximated with the Cartesian forms. Note that a breaker-induced flow separation shown in Fig. 1b appears as Fig. 1c in the surface-fitted coordinate system of our LES. In Eqs. (1) and (3), the regular SGS terms (viz., $R_{i j}, T^{\mathrm{SGS}}$, and $\epsilon$ ) and the breaker effect terms (viz., $\bar{A}_{i}^{m}$ and $W^{m}$ ) require modeling. The regular SGS terms are modeled using a conventional TKE-closure SGS parameterization described by Moeng (1984). Some LES runs are repeated using another TKE-closure SGS parameterization described by Sullivan et al. (1994) in order to investigate the sensitivity of our results to different SGS parameterizations. In both SGS parameterizations, the SGS stress is modeled with eddy viscosity $\nu_{T}$ diagnosed based on $e ; T^{\mathrm{SGS}}$ is modeled as downgradient diffusion of $e$, namely $\left(\partial / \partial x_{j}\right)\left(2 \nu_{T} \partial e / \partial x_{j}\right) ; \epsilon$ is assumed to be proportional to $e^{3 / 2}$. Modeling for the breaker effect terms is described next.

The momentum input $\bar{A}_{i}^{m}$ is specified in such a way that it models localized forcing and wake production occurring in breaking wave event $m$. When wind blows over and around a breaker, a localized pressure perturbation appears at the air-sea interface and in the interior of the air surrounding the breaker. This pressure perturbation at the air-sea interface causes the form drag acting on the breaker. The net pressure gradient force on the surrounding interior airflow takes energy and momentum away from the mean wind and gusts. The aim of $\bar{A}_{i}^{m}$ is to apply this breaker forcing in our LES and to induce energy transfer from the mean wind and gusts to the wake turbulence.

For this reason, $\bar{A}_{i}^{m}$ is defined in the following manner. First, we estimate the form drag acting on a cross section of breaker $m$ (Fig. 1a) based on a conventional aerodynamic drag formula (e.g., Kukulka et al. 2007):

$$
F_{i}^{m}=\rho 2 a C_{d}^{\mathrm{BR}}\left|\mathbf{u}^{\mathrm{AT}}-\mathbf{c}\right|\left(u_{i}^{\mathrm{AT}}-c_{i}\right)
$$

where $F_{i}^{m}$ is the form drag (per unit breaker crest length) acting on the cross section, $\rho$ is the air density, $a$ is the
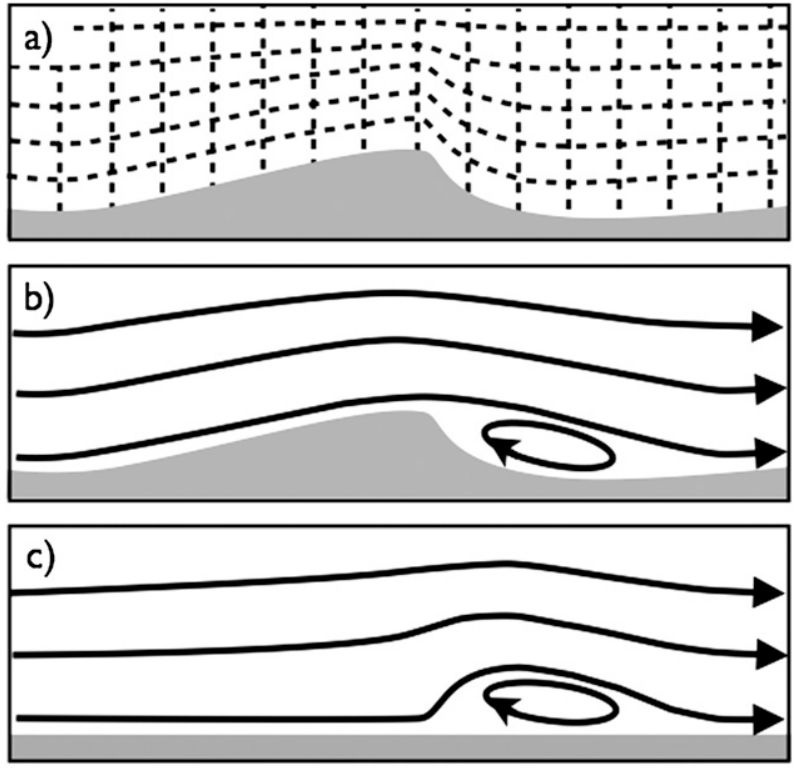

d)

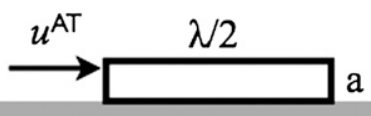

FIG. 1. Schematic explaining the LES approach: (a) cross section of a breaker and a surface-fitted coordinate system around it; (b) rough sketch of an airflow around a breaker; (c) the same airflow as in (b) but seen in the surface-fitted coordinate system in (a); and (d) the box area where $\bar{A}_{i}^{m}$ appears in the LES and the position of the upstream wind used to diagnose the form drag on the breaker.

amplitude of the breaker, $C_{d}^{\mathrm{BR}}$ is an empirically determined form drag coefficient of the breaker, $\mathbf{c}$ is the propagation velocity of the breaker and is assumed to be related to the wavenumber $k$ and the gravitational acceleration $g$ by $c=\sqrt{g / k}$, and $\mathbf{u}^{\mathrm{AT}}$ is a measure of the wind forcing on the breaker cross section. Specifically, $\mathbf{u}^{\mathrm{AT}}$ is set to the instantaneous upstream wind normal to the breaker crest and is parallel to $\mathbf{c}$ located at $z=$ $a$ away from the surface. If $\mathbf{u}^{\mathrm{AT}}$ is opposite to or slower than $\mathbf{c}$, then $F_{i}^{m}$ is set to zero. In this study, we assume that the breaker slope $a k$ is 0.3 for all breakers (i.e., $a$ is set equal to $0.3 / k$ in our simulations). Note that the range of $a k$ is generally confined between 0.1 and 0.5 (Kukulka et al. 2007). Next, we apply the same drag force (with an opposite sign) to the airflow. The drag force is uniformly distributed inside an empirically determined area $V^{m}$ such that $\bar{A}_{i}^{m}$ inside the area is

$$
\bar{A}_{i}^{m}=\frac{-F_{i}^{m}}{\rho V^{m}} .
$$

Outside the area, $\bar{A}_{i}^{m}=0$. 

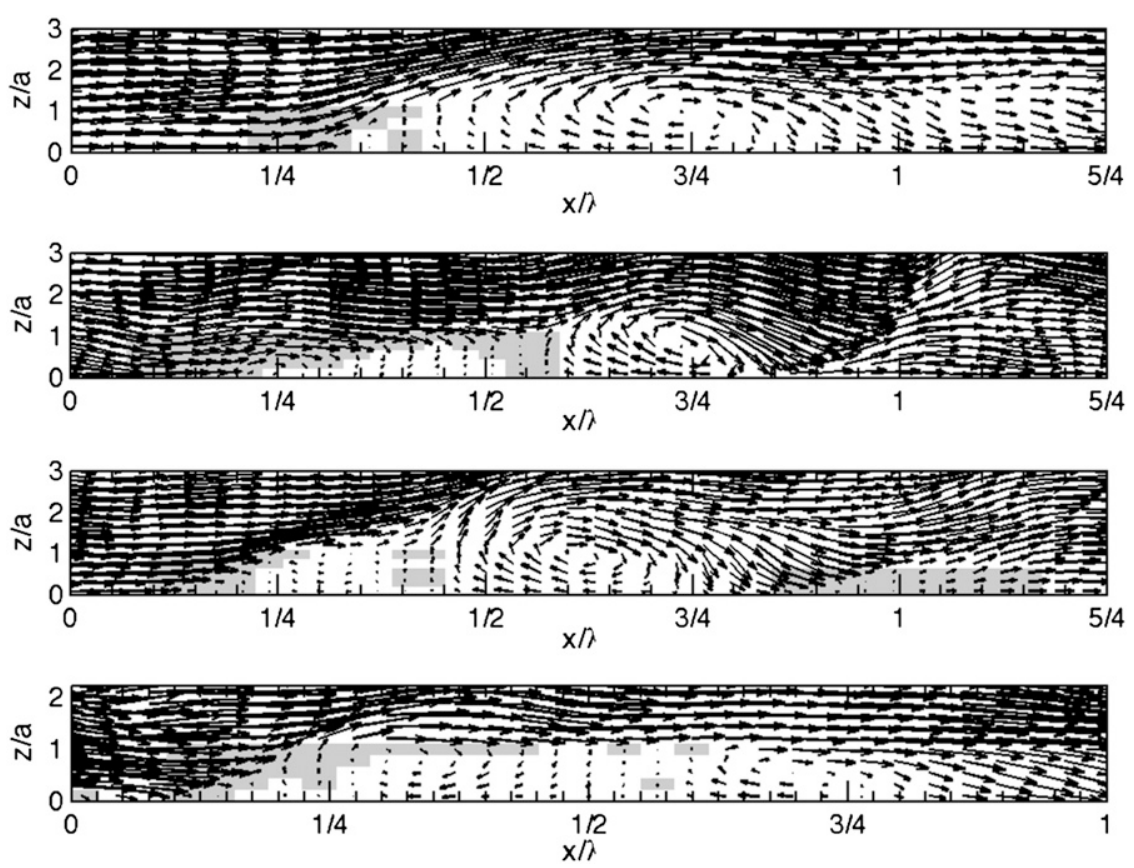

FIG. 2. Examples of wakes induced by breaker forcing in our LES. The breaker forcing appears in the gray areas. Arrows are wind speed vectors minus the propagation speed of the breaker $c$. Height and streamwise length is normalized by the breaker amplitude $a$ and wavelength $\lambda$, respectively.

The form drag coefficient $C_{d}^{\mathrm{BR}}$ in Eq. (4) and the area $V^{m}$ are empirically determined so that the wakes produced in LES are comparable with the breaker-induced wakes observed in the laboratory experiment by Reul et al. (2008). Reul et al. find the following wake characteristics.

1) The wakes are unstable and transient.

2) Often there are multiple recirculation vortices in a flow separation bubble.

3) Often an upward burst of air motion is induced near the downstream side of a reattachment point.

4) Generally, the degree of flow separation depends on the wind forcing intensity and the type of breakers such as microbreaking, spilling, and plunging breaker.

5) The maximum backflow speed can reach about $20 \%$ to $30 \%$ of the mean wind speed at the crest height (i.e., approximately $20 \%$ of the free stream wind speed in their tank).

6) The height of the separation bubble is about the height of the breaker amplitude to the breaker height, and the downwind extent of the separation bubble is about $30 \%$ to $100 \%$ of the wavelength for breakers whose $a k$ is about 0.3 .

After exploring different forcing configurations, we have found that the intensity of the recirculation vortices in our LES is mainly controlled by $C_{d}^{\mathrm{BR}}$, that the size of the separation bubbles is mainly controlled by $V^{m}$, and that the above wake characteristics are well reproduced when the form drag coefficient $C_{d}^{\mathrm{BR}}$ is in the range $0.6<C_{d}^{\mathrm{BR}}<3.0$, and the area $V^{m}$ is a rectangle as shown in Fig. $1 \mathrm{~d}$, where $\lambda=2 \pi / k$ is the wavelength of the breaker. In addition, at the locations where $\left(c_{i}-\bar{u}_{i}\right) \bar{A}_{i}^{m}$ is negative, $\bar{A}_{i}^{m}$ is reset to 0 . This is done to avoid an unphysical (negative) value for the SGS work input $W^{m}$, as explained later. In reality, as the intensity and geometry of a breaker are variable and transient, $C_{d}^{\mathrm{BR}}$ and $V^{m}$ are likely variable and transient as well. However, when we use a static and constant $C_{d}^{\mathrm{BR}}$ and a static $V^{m}$ whose scale is proportional to the breaking wavelength, the simulated wake turbulence is sufficiently unsteady and variable to resemble the foregoing wake characteristics observed by Reul et al. (2008). Introducing variability and unsteadiness in $C_{d}^{\mathrm{BR}}$ or $V^{m}$ does not change our overall results. Figure 2 shows examples of resolvedscale wakes produced in our LES. In the following, we set the baseline (default) value of $C_{d}^{\mathrm{BR}}$ to be 1.0 and use different values of $C_{d}^{\mathrm{BR}}$ (viz., 0.6 and 3.0) only when we investigate the sensitivity of our $C_{D 10}$ results to $C_{d}^{\mathrm{BR}}$.

Finally, modeling for $W^{m}$ in Eq. (3) is done in such a way that the overall energy transfer occurring in a breaking wave event is conserved; that is, the rate of energy loss in the resolved wind is balanced by the rate of energy gain in the SGS turbulence and the rate of energy 
transfer to the breaker. According to Eq. (1), the rate of total work done by breaker forcing on resolved winds in breaking wave event $m$ is given by $\int \bar{u}_{i} \bar{A}_{i}^{m} d x d y d z$ where the integral is taken over the region forced by the breaker. On the other hand, the rate of energy transfer to the breaker may be estimated by the breaker propagation velocity times the form drag, namely $-c_{i} \int \bar{A}_{i}^{m} d x d y d z$ (e.g., Kukulka et al. 2007). Then, the conservation of energy may be written as $\int\left(W^{m}-\right.$ $\left.c_{i} \bar{A}_{i}^{m}+\bar{u}_{i} \bar{A}_{i}^{m}\right) d x d y d z=0$. To satisfy the energy conservation, we simply model $W^{m}$ as $W^{m}=\left(c_{i}-\bar{u}_{i}\right) \bar{A}_{i}^{m}$ in this study. Note that the SGS wake production $W^{m}$ represents the energy transfer to SGS motions from resolved motions. It is unphysical if this term is negative (i.e., if SGS motions convert into large-scale motions by breakers). Therefore, when $W^{m}=\left(c_{i}-\bar{u}_{i}\right) \bar{A}_{i}^{m}$ is locally negative, we set $\bar{A}_{i}^{m}=0$ there.

\section{b. Wave age and average air-sea momentum flux considered}

In this study, we consider wave conditions that appear in a wind-wave tank at high winds. The reasons are threefold. First, in such conditions waves are narrow banded; that is, the entire range of breaking waves can be explicitly considered using computational domains of reasonable size and resolution. Second, we will find that the total momentum flux (wind stress) is mostly supported by the breaking wave form drag, and the contributions from the surface viscous stress and the nonbreaking wave form drag are practically negligible. Third, in the laboratory conditions the drag coefficient $C_{D 10}$ is accurately known and can be compared with the LES results. (In contrast, the drag coefficient in the open ocean is still poorly constrained.) To the best of our knowledge, the wind-wave tank experiment by Donelan et al. (2004) is the most comprehensive experiment at hurricane-strength winds. Their results show the average air-sea momentum flux and the corresponding $U_{10}$ or equivalently $C_{D 10}$. In addition, they report the peak wave frequency at the highest wind speed used in their experiment. The peak wave frequency and average airsea momentum flux can be used to compute the wave age $c_{p} / U_{*}$, where $c_{p}$ is the phase speed at the peak wave frequency.

We perform simulations at two conditions reported in Donelan et al. (2004): 1) $c_{p} / U_{*}=0.5$ and $U_{*}=2.0 \mathrm{~m} \mathrm{~s}^{-1}$ and 2) $c_{p} / U_{*}=0.4$ and $U_{*}=2.65 \mathrm{~m} \mathrm{~s}^{-1}$. The corresponding $U_{10}$ in Donelan et al. is about 40 and $53 \mathrm{~m} \mathrm{~s}^{-1}$, respectively. The former is the condition where $C_{D 10}$ starts to saturate in their experiment, and the latter corresponds to their highest wind speed. Note that the wave age of the first condition is an estimate since the peak wave frequency is not reported at this wind condition.
The estimation is made using an empirical relationship between wave age (or inverse dimensionless peak wave frequency) and dimensionless fetch: namely, $c_{p} / U_{*} \propto$ $\left(X_{f} g / U_{*}^{2}\right)^{\alpha}$ in which $X_{f}$ is the fetch and $\alpha$ is a constant ranging $0.23<\alpha<0.33$ (Babanin and Soloviev 1998).

\section{c. Field of breakers}

During our LES runs, discrete breaking wave events over a range of wavenumbers are generated intermittently in time, randomly in space, and independently from the airflow. Once generated, each breaking wave event lasts for one wave period $2 \pi / \sqrt{g k}$, and its position moves at its breaker propagation velocity c. The spanwise dimension of each breaking wave event is set to its wavelength. These parameter choices follow Sullivan et al. (2007) and Suzuki et al. (2011), and our results are relatively insensitive to the particular choices made here. A random number of breaking wave events at each wavenumber are initiated at each time step in such a way that the resultant breaker field satisfies a specified breaking wave distribution function $\Lambda(k, \sigma)$ on a long time average over the entire bottom boundary. Here, $\sigma$ is the breaker propagation direction, and $\Lambda(k, \sigma) k d k d \sigma$ represents the average length of breaking crests per unit horizontal area of the sea surface for waves with wavenumbers between $k-d k / 2$ and $k+d k / 2$ and propagation directions between $\sigma-d \sigma / 2$ and $\sigma+d \sigma / 2$ (e.g., Phillips 1985; Kleiss and Melville 2011).

Unfortunately, there are scant experimental observations of $\Lambda$ in hurricane-strength winds. Thus, we specify $\Lambda$ based on the Reynolds-averaged Navier-Stokes wave boundary layer model of Kukulka and Hara (2008b). Their RANS model is based on the conservation of wave energy as well as the conservation of airflow momentum and energy, and it predicts $\Lambda$ for fully developed airflow turbulence over very young to mature seas. The predicted $\Lambda$ is consistent with existing observations in open ocean conditions at low to moderate winds where the wave age is 10 or larger. In higher wind speeds and younger sea states, the model results have not been validated as direct observations of $\Lambda$ are not readily available. Therefore, we consider a wide range of uncertainty in $\Lambda$.

According to their RANS model, the directional spreading of $\Lambda$ becomes narrower for younger seas, and it becomes unidirectional in the asymptotic limit of very young sea states. Thus, in the following we assume unidirectionality; that is, we assume that all breaking waves propagate in the mean wind direction. (We tested different directional spreading cases and found that the results are relatively insensitive to this choice.)

Their RANS results strongly depend on several key parameters (viz., the breaker form drag coefficient, the wave energy dissipation rate due to breaking, the breaker 


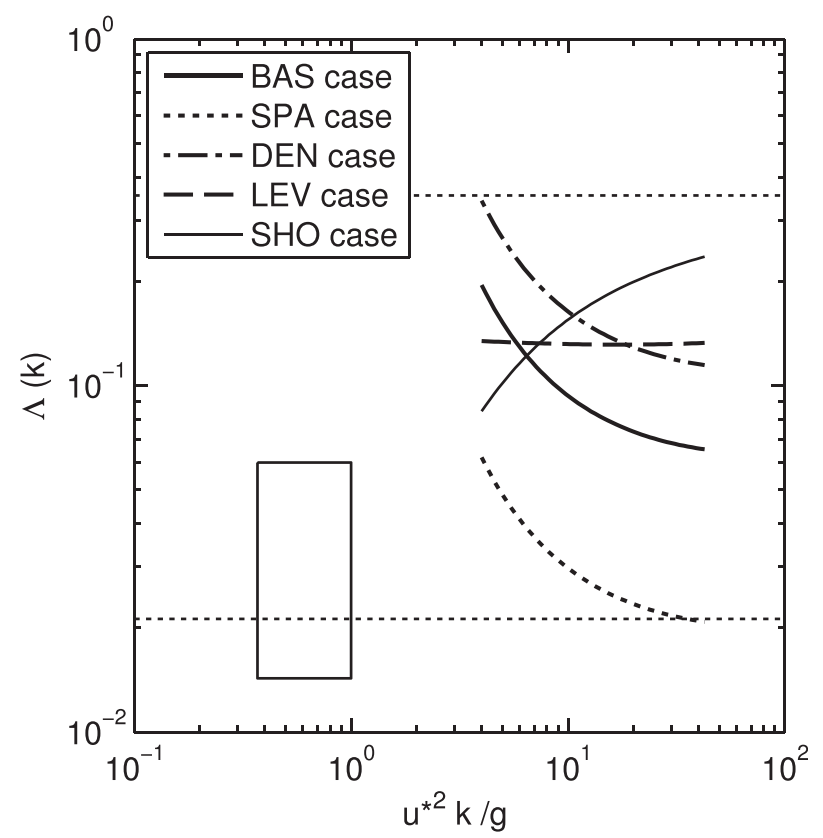

FIG. 3. Breaking distribution $\Lambda(k)$ used in our LES at wave age 0.5: baseline (BAS), sparse (SPA), dense (DEN), level (LEV), and short-breaker dominating (SHO) cases. The horizontal dot lines are the estimated upper and lower bounds of $\Lambda(k)$ (see the appendix). The box shows the estimated range of $\Lambda(k)$ in the laboratory measurement of dominant microbreakers by Jessup and Phadnis (2005).

wave height, and the sheltering coefficient). Since these parameters are not well constrained, the magnitude and shape of $\Lambda(k)$ are also not well constrained. For example, $\Lambda(k)$ may monotonically increase or decrease with $k$. We therefore test several different breaking wave distribution functions. Figure 3 shows the $\Lambda(k)$ used in our simulations at wave age 0.5 . Here, the baseline case (BAS) is determined such that 1$)$ the $\Lambda(k)$ value is between our estimates of the upper and lower bounds described in the appendix, and 2) the $\Lambda(k)$ is the largest for the dominant waves, which we believe is qualitatively consistent with laboratory observations (Jessup and Phadnis 2005). We then investigate the dense (DEN) breaker case and the sparse (SPA) breaker case without altering the $k$ dependence, and level (LEV) and shortbreaker dominating (SHO) cases without altering the overall level of $\Lambda(k)$. In our simulations at wave age 0.4 , the same $\Lambda(k)$ forms have been shifted horizontally such that the peak (i.e., smallest) $u^{* 2} \mathrm{k} / \mathrm{g}$ is located at $6.25 \mathrm{in}$ stead of 4 .

In all simulations, the largest wavenumber of breaking wave events is fixed at $k=88.2 \mathrm{rad} \mathrm{m}^{-1}$ (i.e., wavelength $\lambda=0.071 \mathrm{~m}$ ). Note that a modest change in this cutoff wavenumber does not change our results. In this study, breaking wave events of different scales are allowed to overlap. Figure 4 shows snapshots of the areas where nonzero $\bar{A}_{i}^{m}$ exists at different heights for the three cases of SPA, BAS, and DEN.

\section{d. Numerical method}

Time integration uses an explicit, third-order, threesubstep Runge-Kutta scheme. A fixed time step is used based on a fixed Courant-Fredrichs-Lewy condition $\left(\Delta t U_{*} / a_{o}=0.02\right.$ or 0.015 depending on the simulated cases). Horizontal differentiation uses the pseudospectral method. Vertical differentiation uses the secondorder centered finite difference method on a vertically staggered grid. The variables $\bar{w}, e$, and $W^{m}$ are stored at the same grid levels (hereafter, $w$-nodes), and $\bar{u}, \bar{v}, \bar{p}$, $\bar{A}_{1}^{m}$, and $\bar{A}_{2}^{m}$ are stored at the grid levels (hereafter, $u$-nodes) located midway between the $w$-nodes. The $w$-nodes hold the bottom and top boundaries. The bottom boundary is at $z=0$. The grid is horizontally uniform and vertically nonuniform. We locate the fifth $u$ node at $z=a_{o}$ where $a_{o}$ is the amplitude of the tallest breaker, and set the distances of the lowest six $w$ spacing to be $\Delta z / a_{o}=2 / 9$. Above this, each $w$ spacing $\Delta z / a_{o}$ is 1.03 times larger than the spacing one-node below.

The horizontal boundaries are periodic. The top boundary is frictionless and nonpermeable. The bottom boundary is nonpermeable. For the bottom SGS stress, we tested several different parameterizations including a conventional one (Moeng 1984) and find that our results are relatively insensitive to a modest change in bottom SGS stress parameterizations. This is because breaker forcing is responsible for almost the entire airsea momentum flux, and the mean wind near the water surface is small in all simulations presented in this paper.

The horizontal domain size $L_{x} \times L_{y}$ is $L_{x} / a_{o}=L_{y} / a_{o}=$ 83.78; $L_{x}$ is four times the wavelength of the largest breaking wave considered. The domain height $L_{z}$ is $L_{z} / a_{o}=56.22$. The grid has $128 \times 128$ nodes horizontally and 96 nodes vertically.

The initial condition is a small and uniform streamwise wind everywhere. All results are obtained after the flow is converged to a statistically steady (i.e., fully developed) state. Note that, in the current LES, the breaker field $(\Lambda)$ is kept constant in time and space (i.e., the wave growth in time or space is ignored). In reality, the wave field evolves in time or space at real young sea conditions; as a result, the airflow turbulence in such conditions may not be horizontally homogeneous nor steady. However, in this study, we assume that airflow at young sea conditions may be approximated with the horizontal periodicity and fully developed state of airflow turbulence.

Some quantities are averaged for the following analysis. The averaging is done over a horizontal plane and over a long time (i.e., 71 large-eddy turnover time $t U_{*} / L_{z}$ ). 

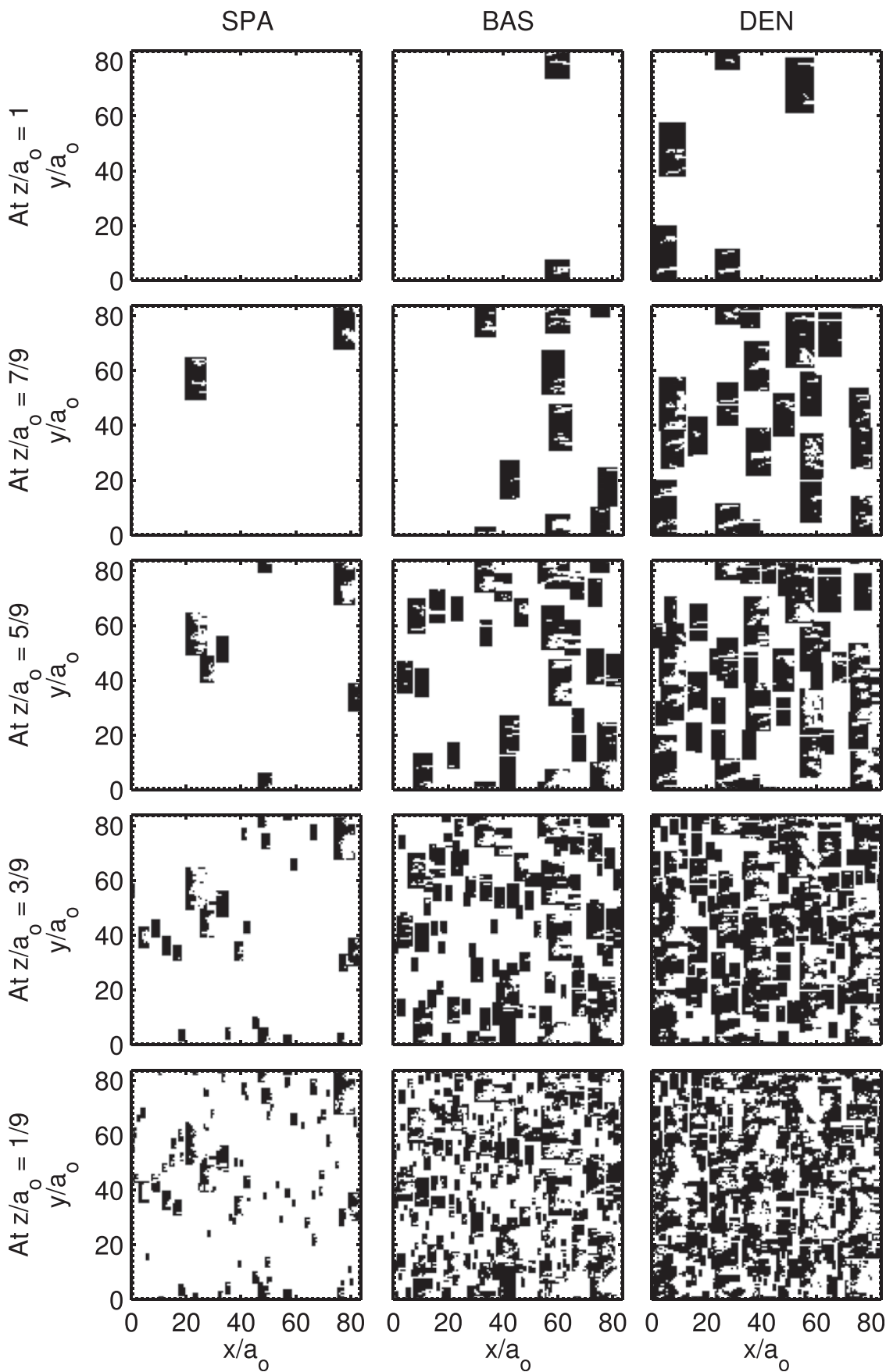

FIG. 4. Snapshots of instantaneous areas (black) where breaker forcing appears at different heights: (left) SPA case, (middle) BAS case, and (right) DEN case.

\section{Results of low-order moments}

\section{a. Mean wind profile and drag coefficient}

In the following, angle brackets denote a horizontal average, and a single prime denotes the deviation from it; for example, $\bar{u}=\langle\bar{u}\rangle+\bar{u}^{\prime}$. First, let us investigate the mean wind profiles. Figure 5 shows the normalized mean wind shear $\phi_{m} \equiv\left(z \kappa / U_{*}\right) d\langle\bar{u}\rangle / d z$ at wave age $c_{p} / U_{*}=$ 0.5. In the figure, the distance from the water surface is normalized with the amplitude of the tallest breaker $a_{o}$. The results at $c_{p} / U_{*}=0.4$ are not shown since they are essentially identical to the ones shown. The breaker conditions tested are five cases of different $\Lambda$ (BAS, DEN, SPA, LEV, SHO; see Fig. 3) with the default breaker 


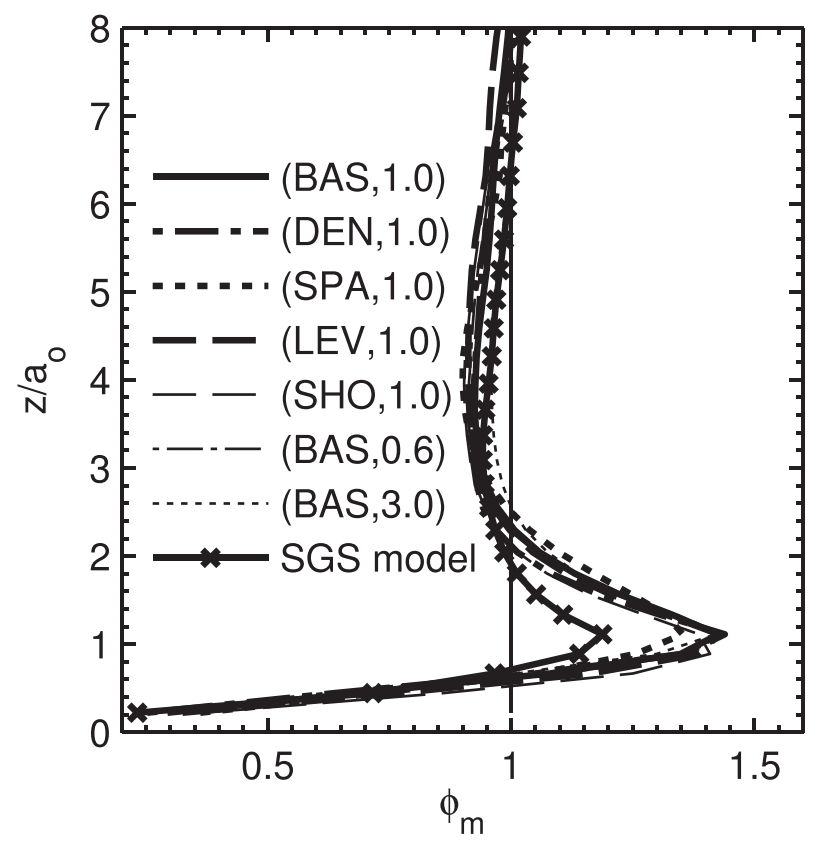

FIG. 5. Vertical profiles of normalized wind shear $\phi_{m}$ at wave age $=0.5$ and $U_{*}=2 \mathrm{~m} \mathrm{~s}^{-1}$. For a logarithmic wind profile $\phi_{m}=1$ at every height. The legend shows $\Lambda(k)$ and $C_{d}^{\mathrm{BR}}$ value for each case. See Fig. 3 for the definitions of the $\Lambda(k)$ value/shape. The solid cross-marked line is obtained using the Sullivan SGS model with the baseline (BAS) $\Lambda$ and $C_{d}^{\mathrm{BR}}=1.0$.

form drag coefficient $\left(C_{d}^{\mathrm{BR}}=1\right)$ and two cases of different $C_{d}^{\mathrm{BR}}(=0.6$ and 3.0) with the baseline $\Lambda$. In addition, one run (BAS $\Lambda$ with $C_{d}^{\mathrm{BR}}=1$ ) is repeated using a different SGS model by Sullivan et al. (1994). The results show little dependence on the different breaker conditions and only weak dependence on the choice of SGS models (near $z / a_{o}=1$ ). Thus, the impacts of breakers are robust and not significantly affected by the uncertainties in $\Lambda$ and $C_{d}^{\mathrm{BR}}$ or the different SGS models.

Away from the surface, the wind profiles are logarithmic (i.e., $\phi_{m}=1$ ) as expected; the profiles are roughly logarithmic above $2 a_{o}$ and nearly perfectly logarithmic above $5 a_{o}$ to $6 a_{o}$. This height of the log-layer bottom is similar to turbulent flows over other types of roughness (e.g., Ikeda and Durbin 2007). In contrast, the wind profiles are not logarithmic (i.e., $\phi_{m} \neq 1$ ) near the surface in the WBL. The solutions show the existence of three characteristic regions in the atmospheric wave boundary layer: 1) the region well inside the WBL where the wind shear is much less than the log-profile shear, 2) the region near the top of the WBL around $z / a_{o}=1$ where the shear is higher than the log-profile shear, and 3 ) the region around $2 \leq z / a_{o} \leqq 5$ where the shear is slightly lower than the log-profile shear.

In the first and second regions, the mean wind is not logarithmic because of the breaker-induced wakes. When the flow separates over a breaker, the region of very high shear that is usually attached on the water surface separates from the surface and appears along the edge of the separation bubble (Fig. 6). Hence, the wind profile spatially averaged at the separation bubble height (i.e., the second region) becomes steeper than the logarithmic wind profile. On the other hand, the local wind shear inside the separation bubble is much lower than the log-profile shear (Fig. 6). Hence, the spatially averaged wind profile well below the separation bubble height (i.e., the first region) is less steep than the logprofile. The same shear patterns of breaker-induced wakes are also shown in the particle image velocimetry (PIV) images by Reul et al. (2008). In addition, a similar trend of the mean wind shear is observed in the direct numerical simulation (DNS) over $k$-type roughness (Ikeda and Durbin 2007).

In the third region $\left(2 \lesssim z / a_{o} \lesssim 5\right)$, the shear is slightly lower because the breaker forcing is anisotropic (Suzuki et al. 2011). At young sea states, breaking waves appear mostly perpendicular to the mean wind. Because the pressure form drag is normal to the breakers, the breaker forcing is mostly streamwise, and spanwise turbulent winds experience little drag. Such anisotropic drag results in reduced dissipation of the surface-attached log-layer quasi-streamwise vortices. The enhanced quasistreamwise vortices, then, result in increased vertical mixing and reduced wind shear (and associated reduced TKE shear production).

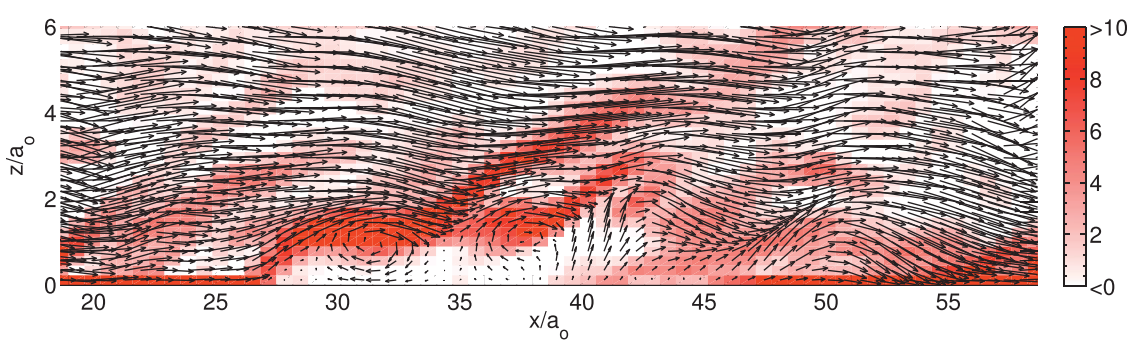

FIG. 6. Example of high-shear region around breaker-induced flow separation. The color shows $d \bar{u} / d z$ normalized by $U^{*} / a_{o}$. 

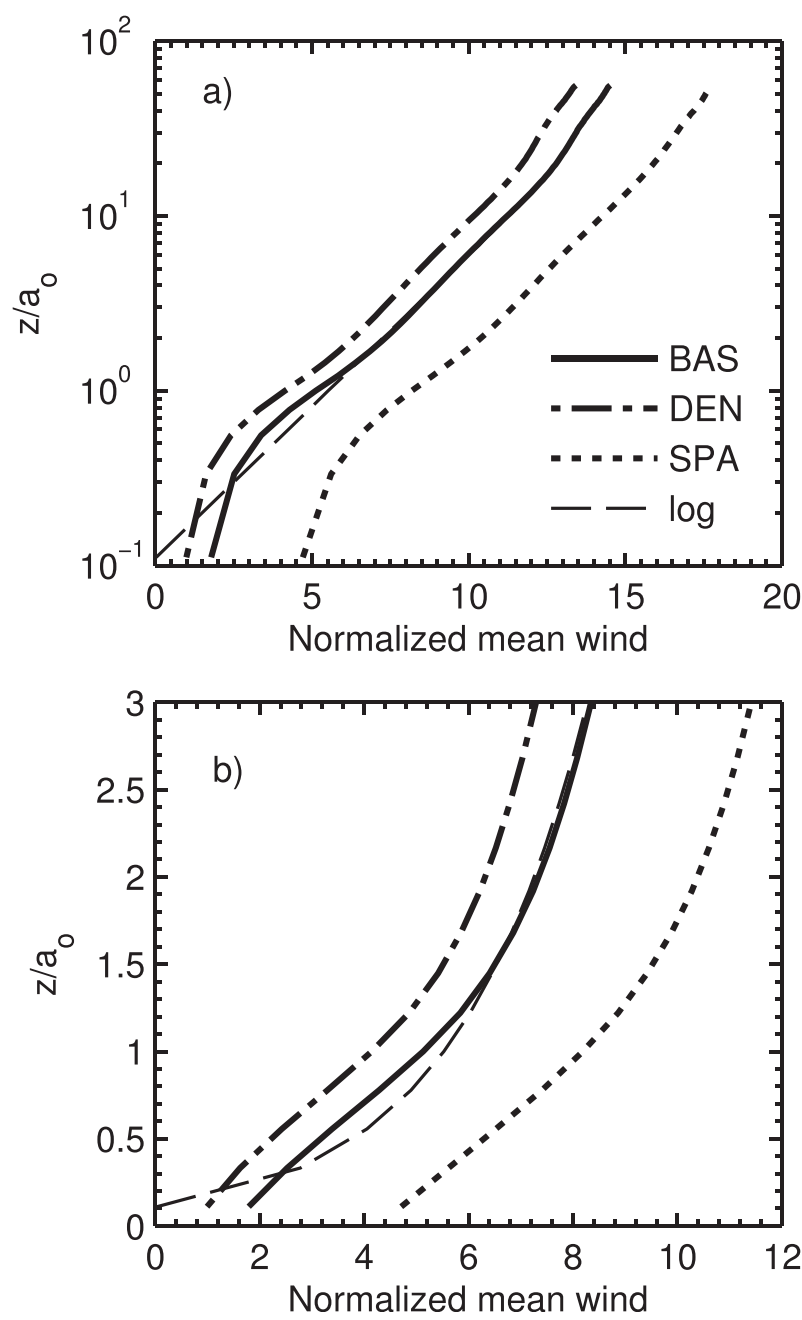

FIG. 7. Vertical profiles of normalized mean wind speed $\langle\bar{u}\rangle / U_{*}$ : (a) entire profile on a log-linear axis and (b) near-surface part on a linear-linear axis. The legend of (b) is the same as that of (a). Cases shown are with wave age $=0.5, U_{*}=2 \mathrm{~m} \mathrm{~s}^{-1}, C_{d}^{\mathrm{BR}}=1.0$, and three different $\Lambda$ (DEN, BAS, and SPA). For reference, a logprofile with $C_{D 10}=0.0025$ is also shown.

In summary, the WBL wind profile is not analogous to the wind profile over a flat wall. It is strongly modified due to the breaker-induced flow separation (first and second regions) and, to a much less extent, the directionality of the breakers (third region). Figure 7 shows the mean wind profiles for the three cases having different breaker densities (DEN, BAS, and SPA with $\left.C_{d}^{\mathrm{BR}}=1.0\right)$. Notice that the overall change in breaker density affects the mean wind speed (and the drag coefficient) even if it hardly affects the mean wind shear.

Next, we show the drag coefficient $C_{D 10}$ (Fig. 8). It is computed from the mean wind speed in the log layer above the WBL. Overall, $C_{D 10}$ falls in the range between 0.002 and 0.003 . If the breaker distribution is kept

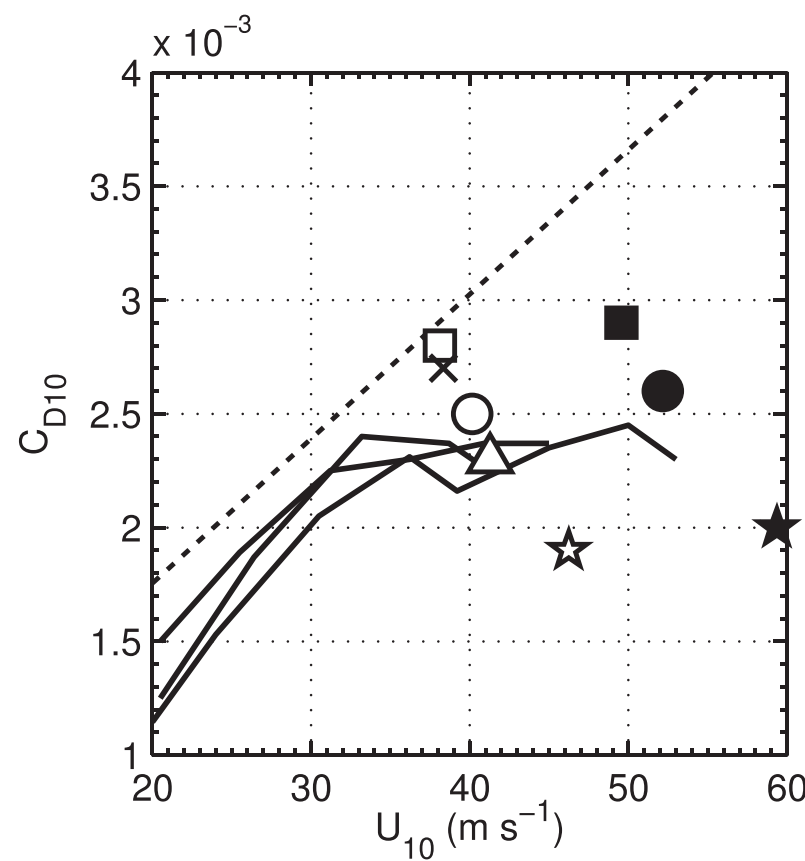

FIG. 8. Drag coefficient vs wind speed. All symbols are our LES results. Open symbols are at wave age 0.5 and $U_{*}=2 \mathrm{~m} \mathrm{~s}^{-1}$, and the solid symbols are at wave age 0.4 and $U_{*}=2.65 \mathrm{~m} \mathrm{~s}^{-1}$. Results with a fixed $C_{d}^{\mathrm{BR}}=1.0$ and different levels of $\Lambda$ are shown by squares (DEN), circles (BAS), and stars (SPA). Results with a fixed $\Lambda$ (BAS) and different values of $C_{d}^{\mathrm{BR}}$ are shown by a cross $\left(C_{d}^{\mathrm{BR}}=3.0\right)$, a circle $\left(C_{d}^{\mathrm{BR}}=1.0\right)$, and a triangle $\left(C_{d}^{\mathrm{BR}}=0.6\right)$ at wave age 0.5 only. Solid lines are laboratory experimental results shown in Donelan et al. (2004). Dotted line is bulk formula by Large and Pond (1981).

roughly the same as the wind speed increases, then the $C_{D 10}$ remains nearly constant at high winds. As the overall breaker density increases (DEN) or decreases (SPA) compared to the baseline case (BAS), the drag coefficient increases or decreases as expected: $C_{D 10}$ increases by about $50 \%$ when the amount of breaking events increases by about sixfold (from SPA to DEN). If the breaker form drag coefficient $C_{d}^{\mathrm{BR}}$ increases or decreases, the drag coefficient $C_{D 10}$ also increases or decreases, but the impact is smaller. We also find that varying the $k$ dependence of $\Lambda$ (LEV and SHO cases compared to BAS case) has negligible effects on the drag coefficient (not shown). The effect of varying $\Lambda$ at wave age $c_{p} / U_{*}=0.5$ and $U_{*}=2.0 \mathrm{~m} \mathrm{~s}^{-1}$ (open symbols) is almost identical to that at wave age $c_{p} / U_{*}=0.4$ and $U_{*}=2.65 \mathrm{~m} \mathrm{~s}^{-1}$ (solid symbols). In summary, our LES results of $C_{D 10}$ are roughly consistent with laboratory observations although the large uncertainties in $\Lambda$ and $C_{d}^{\mathrm{BR}}$ yield $C_{D 10}$ varying between 0.002 and 0.003 .

Since the overall results are not overly sensitive to $C_{d}^{\mathrm{BR}}$ or the $k$ dependence of $\Lambda$, we examine only the three cases, namely DEN, BAS, and SPA, with $C_{d}^{\mathrm{BR}}=1.0$ hereafter. 


\section{b. Energy budget of the WBL}

The energy budget of the WBL provides valuable insight into why $C_{D 10}$ saturates in high winds. Let $E_{\mathrm{M}}=\left\langle\bar{u}_{i}\right\rangle\left\langle\bar{u}_{i}\right\rangle / 2$ be the kinetic energy of the mean wind and $E_{\mathrm{RT}}=\bar{u}_{i}^{\prime} \bar{u}_{i}^{\prime} / 2$ be the TKE of the resolved-scale turbulence. According to Eq. (1) and Eq. (3), in a statistically steady state, the energy budgets of the mean flow, the resolved-scale TKE, and the SGS TKE can be expressed as

$$
\begin{aligned}
0=\frac{\partial\left\langle E_{\mathrm{M}}\right\rangle}{\partial t}= & -\frac{\partial\langle\bar{u}\rangle\left\langle\bar{u}^{\prime} \bar{w}^{\prime}+\bar{R}_{13}\right\rangle}{\partial z}+\underbrace{\left\langle\bar{R}_{13}\right\rangle \frac{\partial\langle\bar{u}\rangle}{\partial z}}_{-P_{\mathrm{MS}}^{\mathrm{SGS}}} \\
& +\underbrace{\left\langle\bar{u}^{\prime} \bar{w}^{\prime}\right\rangle \frac{\partial\langle\bar{u}\rangle}{\partial z}-\langle\bar{u}\rangle \frac{\partial \bar{P}}{\partial x}}_{-P_{\mathrm{MS}}^{\mathrm{R}}} \\
& +\underbrace{\left\langle\sum_{m} c_{i}^{m} \bar{A}_{i}^{m}\right\rangle-\left\langle P_{\mathrm{W}}^{\mathrm{R}}\right\rangle-\left\langle P_{\mathrm{W}}^{\mathrm{SGS}}\right\rangle}_{-\langle\bar{u}\rangle\left\langle\sum_{m} \bar{A}_{1}^{m}\right\rangle}, \\
0=\frac{\partial\left\langle E_{\mathrm{RT}}\right\rangle}{\partial t}= & -\frac{\partial}{\partial z}\left(\left\langle\bar{w}^{\prime} E_{\mathrm{RT}}\right\rangle+\left\langle\bar{p}^{\prime} \bar{w}^{\prime}\right\rangle+\left\langle\bar{u}_{i}^{\prime} \bar{R}_{i 3}^{\prime}\right\rangle\right) \\
& +\underbrace{\left\langle R_{i j}^{\prime} \frac{\partial \bar{u}_{i}^{\prime}}{\partial x_{j}}\right\rangle}_{-P_{\mathrm{RTS}}^{\mathrm{SGS}}} \underbrace{-\left\langle\bar{u}^{\prime} \bar{w}^{\prime}\right\rangle \frac{\partial\langle\bar{u}\rangle}{\partial z}}_{P_{\mathrm{MS}}^{\mathrm{R}}}+\underbrace{\left\langle\sum_{m} \bar{u}_{i}^{\prime} \bar{A}_{i}^{m}\right\rangle}_{\left\langle P_{\mathrm{W}}^{\mathrm{R}}\right\rangle},
\end{aligned}
$$

$$
\begin{aligned}
0=\frac{\partial\langle e\rangle}{\partial t}= & -\frac{\partial}{\partial z}\left(\left\langle\bar{w}^{\prime} e^{\prime}\right\rangle-\left\langle 2 \nu_{T} \frac{\partial e}{\partial z}\right\rangle\right)+P_{\mathrm{MS}}^{\mathrm{SGS}} \\
& +P_{\mathrm{RTS}}^{\mathrm{SGS}}+\left\langle P_{\mathrm{W}}^{\mathrm{SGS}}\right\rangle-\langle\epsilon\rangle,
\end{aligned}
$$

where $P_{\mathrm{W}}^{\mathrm{SGS}}=\sum_{m} W^{m}$. Here Eq. (6) is obtained by taking the inner product of $\left\langle\bar{u}_{i}\right\rangle$ and the horizontal average of Eq. (1). Equation (7) is obtained by subtracting Eq. (6) from the horizontal average of the product of $\bar{u}_{i}$ and Eq. (1). [Note that the last term in Eq. (7) can be written as $\left\langle\sum_{m} \bar{u}_{i}^{\prime} \bar{A}_{i}^{m^{\prime}}\right\rangle$, but we prefer the form shown because $\bar{A}_{i}^{m}=0$ and $\bar{A}_{i}^{m^{\prime}} \neq 0$ outside breaking wave events and it allows an easier physical interpretation.] Equation (8) is simply the horizontal average of Eq. (3). In these equations, $P_{\mathrm{MS}}^{\mathrm{R}}$ and $P_{\mathrm{MS}}^{\mathrm{SGS}}$ are the production of resolvedscale and SGS turbulence due to the mean-wind shear $\partial\langle\bar{u}\rangle / \partial z$, respectively, and $P_{\mathrm{RTS}}^{\mathrm{SGS}}$ is the production of SGS turbulence due to the resolved turbulent wind shear $\partial \bar{u}_{i}^{\prime} / \partial x_{j}$. The terms $\left\langle P_{\mathrm{W}}^{\mathrm{R}}\right\rangle$ and $\left\langle P_{\mathrm{W}}^{\mathrm{SGS}}\right\rangle$ are the rate of work done by breaker forcing on resolved scale turbulence and SGS turbulence, respectively.

The energy budget of the total energy $E=E_{\mathrm{M}}+$ $E_{\mathrm{RT}}+e$ and the total TKE $E_{\mathrm{TKE}}=E_{\mathrm{RT}}+e$ can be obtained using Eqs. (6), (7), and (8):

$$
\begin{aligned}
0=\frac{\partial\langle E\rangle}{\partial t}= & -\frac{\partial\langle\bar{u}\rangle\left\langle\bar{u}^{\prime} \bar{w}^{\prime}+\bar{R}_{13}\right\rangle}{\partial z}-\frac{\partial\left\langle f_{T}\right\rangle}{\partial z}-\langle\epsilon\rangle \\
& -\langle\bar{u}\rangle \frac{\partial \bar{P}}{\partial x}+\left\langle\sum_{m} c_{i} \bar{A}_{i}^{m}\right\rangle
\end{aligned}
$$

and

$$
\begin{aligned}
0=\frac{\partial\left\langle E_{\mathrm{TKE}}\right\rangle}{\partial t}= & -\frac{\partial\left\langle f_{T}\right\rangle}{\partial z}+\left(P_{\mathrm{MS}}^{\mathrm{R}}+P_{\mathrm{MS}}^{\mathrm{SGS}}\right) \\
& +\underbrace{\left(\left\langle\sum_{m} c_{i} \bar{A}_{i}^{m}\right\rangle-\langle\bar{u}\rangle\left\langle\sum_{m} \bar{A}_{1}^{m}\right\rangle\right)}_{\left\langle P_{\mathrm{W}}^{\mathrm{R}}\right\rangle+\left\langle P_{\mathrm{W}}^{\mathrm{SGS}}\right\rangle}-\langle\epsilon\rangle,
\end{aligned}
$$

where

$$
f_{T}=\bar{w}^{\prime} E_{\mathrm{RT}}+\bar{p}^{\prime} \bar{w}^{\prime}+\bar{u}_{i}^{\prime} \bar{R}_{i 3}^{\prime}+\bar{w}^{\prime} e^{\prime}-2 \nu_{T} \frac{\partial e}{\partial z}
$$

is the TKE flux. The terms on the rhs of Eq. (10) are called the TKE transport, shear production, wake production, and viscous dissipation, respectively. Note that the fourth term on the rhs of Eq. (9) is the rate of work done by the externally imposed background forcing $-\partial \bar{P} / \partial x$, used to drive the flow. This term does not exist for a turbulent Couette flow and the atmospheric surface layer (i.e., a constant stress layer with no Coriolis effect) since there is no background pressure gradient forcing for these flows. In the current LES, this term is negligibly small in and near the WBL. Hence, our results in and near the WBL are still representative of the energy budget of a constant stress layer.

The relationship between the energy budget and the drag coefficient can be obtained by vertically integrating Eq. (9) from the surface to some height $H_{L}$ inside the log layer and by considering the overall energy budget in this layer. Note that $\langle\bar{u}\rangle$ and $\left\langle f_{T}\right\rangle$ are either zero or very small at the surface. Thus, omitting these terms at the surface as well as the aforementioned small background forcing term, we can express the normalized mean wind speed at $H_{L}$ as

$$
\begin{aligned}
\frac{\langle\bar{u}\rangle\left(z=H_{L}\right)}{U_{*}}= & \frac{\left\langle f_{T}\right\rangle\left(z=H_{L}\right)}{U_{*}^{3}}+\int_{z=0}^{H_{L}} \frac{\langle\epsilon\rangle}{U_{*}^{3}} d z \\
& +\int_{z=0}^{H_{L}} \frac{\left\langle-\sum_{m} c_{i} \bar{A}_{i}^{m}\right\rangle}{U_{*}^{3}} d z .
\end{aligned}
$$

Here the Reynolds shear stress $\left\langle\bar{u}^{\prime} \bar{w}^{\prime}+\bar{R}_{13}\right\rangle$ at $z=H_{L}$ is approximated to be $-U_{*}^{2}$. This substitution is exact for a constant stress layer. The left-hand side represents the downward energy flux (energy input) $\langle\bar{u}\rangle\left\langle\bar{u}^{\prime} \bar{w}^{\prime}+\bar{R}_{13}\right\rangle$ at 


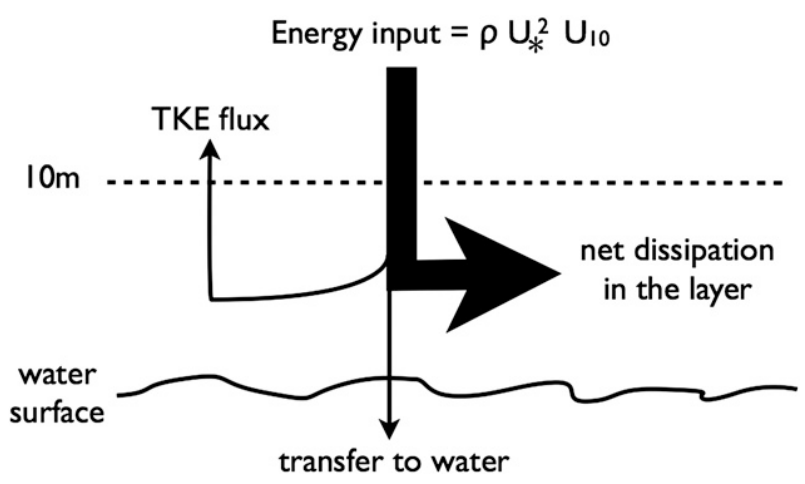

FIG. 9. Schematic showing the energy budget over the layer between 0 to $10 \mathrm{~m}$.

the layer top, normalized by $U_{*}^{3}$ (Fig. 9). This energy input is balanced by the right-hand side representing energy outputs from the layer (Fig. 9): namely, the upward TKE flux at the layer top, the TKE dissipation integrated over the layer, and the energy transfer to the breakers integrated over the layer (all terms normalized by $\left.U_{*}^{3}\right)$. Notice that, by definition, the lhs of Eq. (12) equals $1 / \sqrt{C_{D 10}}$ when $H_{L}=10 \mathrm{~m}$; thus,

$$
\begin{aligned}
C_{D 10}= & \left(\frac{\left\langle f_{T}\right\rangle(z=10 \mathrm{~m})}{U_{*}^{3}}+\int_{z=0}^{10 \mathrm{~m}} \frac{\langle\epsilon\rangle}{U_{*}^{3}} d z\right. \\
& \left.+\int_{z=0}^{10 \mathrm{~m}} \frac{\left\langle-\sum_{m} c_{i} \bar{A}_{i}^{m}\right\rangle}{U_{*}^{3}} d z\right)^{-2} .
\end{aligned}
$$

In summary, Eqs. (12) and (13) show that for a given wind stress the reference wind is higher or the drag coefficient is lower when the surface layer fluxes out or dissipates more energy.

In all cases of this study we find that the integrated energy transfer to the breakers [the third term on the rhs of Eq. (12)] is much less than the integrated TKE dissipation [the second term on the rhs of Eq. (12)]. It is small because the normalized breaker propagation speed $c / U_{*}$ (i.e., the wave age) of the laboratory-scale short waves are very small. Likewise, the TKE flux at the layer top [the first term on the rhs of Eq. (12)] is much less than the integrated TKE dissipation. Therefore, the TKE dissipation is the dominant factor in determining the drag coefficient of very young seas in hurricanestrength winds. Most importantly, the $C_{D 10}$ observed in our LES and the laboratory experiment implies that the normalized TKE dissipation in the surface layer is large and saturates in high winds.

This large TKE dissipation is closely related to the large production of small-scale wake turbulence in the WBL. Figure 10 shows the TKE budget for three cases
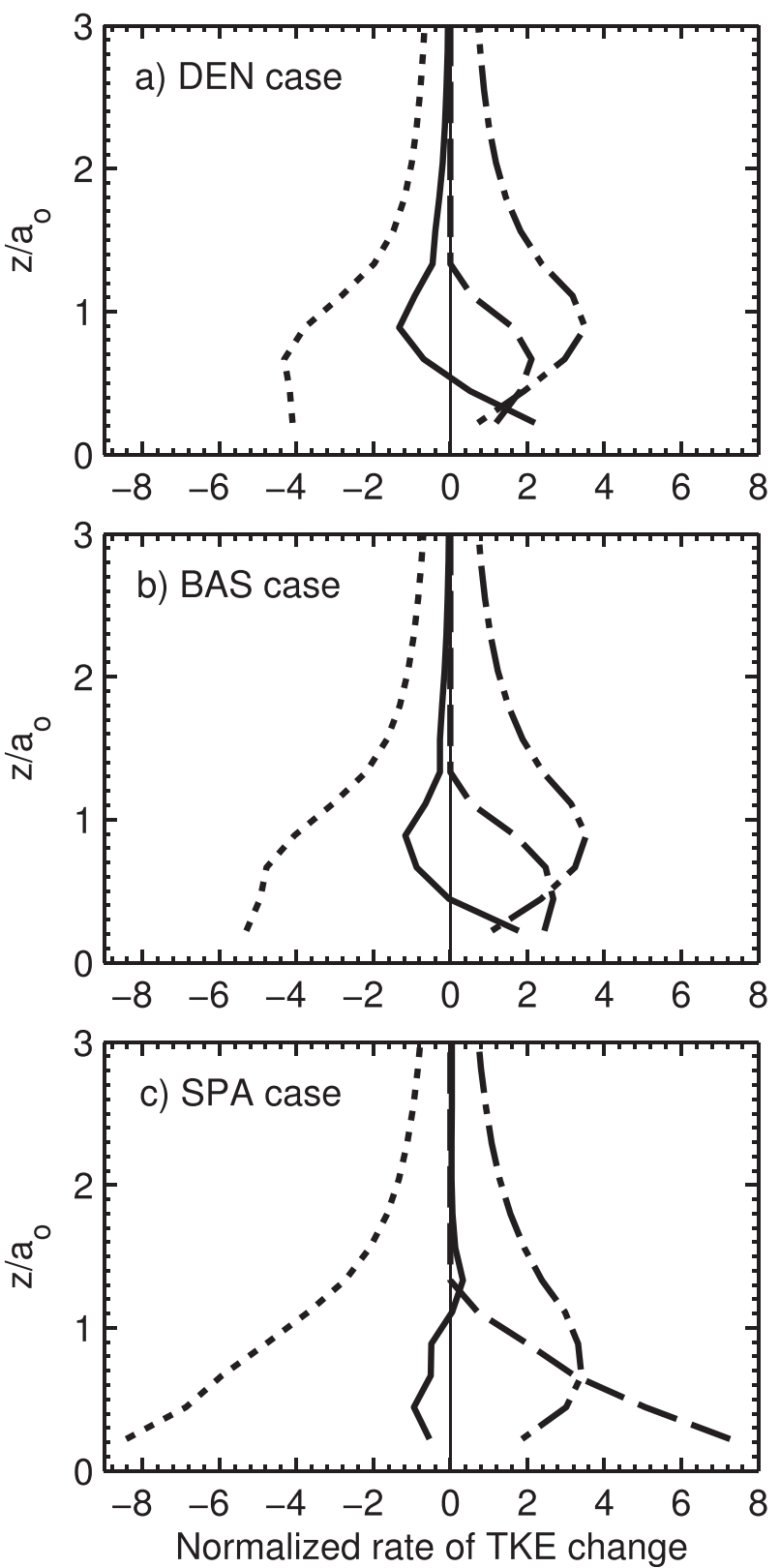

FIG. 10. TKE budget, four terms on the rhs of Eq. (10) normalized by $U_{*}^{3} / a_{o}$ : transport term (solid line), shear production (dashdot line), wake production (dashed line), and dissipation (dotted line). Cases shown are with wave age $=0.5, U_{*}=2 \mathrm{~m} \mathrm{~s}^{-1}$, $C_{d}^{\mathrm{BR}}=1.0$, and three different $\Lambda$ (DEN, BAS, and SPA).

(DEN, BAS, SPA) having different breaker densities. The vertical profiles shown are the four terms on the rhs of Eq. (10), normalized by $U_{*}^{3} / a_{o}$. In all cases, the TKE budget away from the surface $\left(z / a_{o}>2\right)$ is similar to that over flat walls; namely, the shear production balances the dissipation locally at each height. However, this similarity disappears inside the WBL. While the shear production decreases, the wake production due to breakers 
increases significantly and exceeds the shear production in the lower part of the WBL. Because the wake production in high winds is large enough to replace the reduction of the shear production, the net (i.e., the sum of the shear and wake) TKE production stays large and keeps the TKE dissipation large. As a result, $C_{D 10}$ remains small.

While the above statement holds true at any breaker density, the TKE budget also shows a notable dependence on the breaker density. In particular, as the breaker density becomes lower (from DEN to SPA), the wake production and the dissipation become larger (Fig. 10) and the drag coefficient becomes smaller (Fig. 8). The TKE transport also shows some dependence.

\section{c. Validity of existing RANS WBL parameterizations}

As mentioned in the introduction, previous studies of the WBL based on RANS modeling rely on turbulence parameterizations derived by analogy to flat-wall turbulence. We have already seen, in Figs. 5 and 7, that the wind profile inside the WBL is significantly different from the log profile, which is the assumed wind profile in some RANS models. The log-profile assumption overestimates the wind speed near the top of the WBL and significantly underestimates the wind speed in the lower part of the WBL. Therefore, such an assumption may lead to erroneous estimates of the drag coefficient.

The RANS models by Kukulka et al. (2007) and Kukulka and Hara (2008a,b) assume that the TKE dissipation is simply related to the Reynolds shear stress $\left(-\left\langle\bar{u}^{\prime} \bar{w}^{\prime}+\bar{R}_{13}\right\rangle\right)$ as

$$
\langle\epsilon\rangle=\frac{\left(-\left\langle\bar{u}^{\prime} \bar{w}^{\prime}+\bar{R}_{13}\right\rangle\right)^{3 / 2}}{\kappa z}
$$

at each height where $\kappa=0.4$ is the von Kármán constant. In Fig. 11, the TKE dissipation parameterized by Eq. (14) is computed using the LES result of $\left\langle\bar{u}^{\prime} \bar{w}^{\prime}+\bar{R}_{13}\right\rangle$ and is compared to the TKE dissipation resulted in our LES. In all cases, the RANS dissipation model significantly underestimates the TKE dissipation, particularly in the lower part of the WBL. The vertically integrated TKE dissipation is also underestimated appreciably. Therefore, these RANS models likely overestimate the drag coefficient.

Above the layer where Eq. (14) underestimates $\langle\epsilon\rangle$, there is a layer where Eq. (14) overestimates $\langle\epsilon\rangle$ (Fig. $11 \mathrm{~b})$. This is because the RANS parameterization is designed without accounting for the very small, but nonzero, TKE transport (Fig. 10) and the reduction of the wind shear (Fig. 5) in this layer. However, it is clear from Fig. 11a that the overestimation here is not nearly as important as the underestimation below $z / a_{o} \approx 1$.
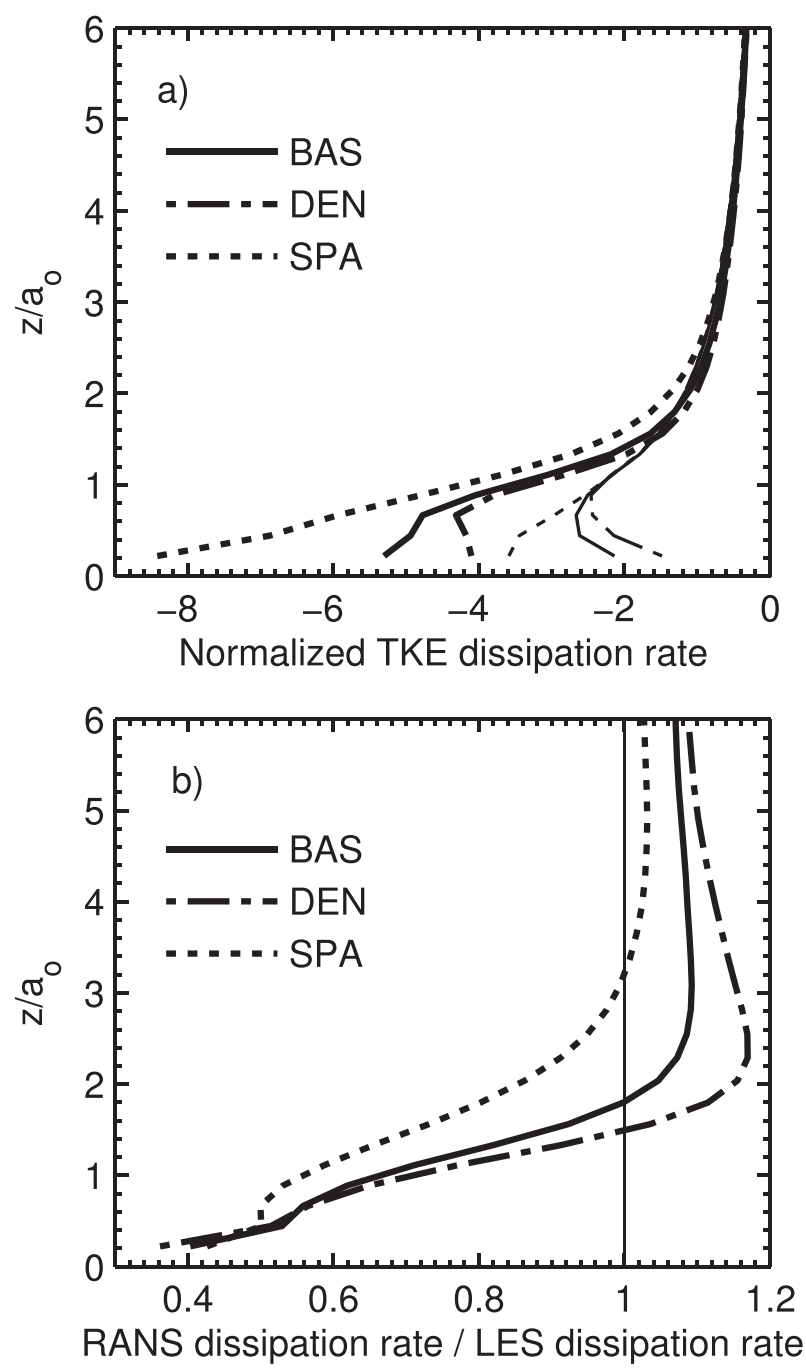

FIG. 11. Comparison of the TKE dissipation rate $\langle\epsilon\rangle$ obtained in the LES and $\langle\epsilon\rangle$ estimated using the RANS parameterization: (a) LES $\langle\epsilon\rangle$ normalized with $U_{*}^{3} / a_{o}$ (thick lines) and RANS $\langle\epsilon\rangle$ normalized with $U_{*}^{3} / a_{o}$ (thin lines). (b) Ratio of the RANS $\langle\epsilon\rangle$ to LES $\langle\epsilon\rangle$. Cases shown are with wave age $=0.5, U_{*}=2 \mathrm{~m} \mathrm{~s}^{-1}$, $C_{d}^{\mathrm{BR}}=1.0$, and three different $\Lambda$ (DEN, BAS, and SPA).

\section{Results of turbulence structures and their characteristics}

\section{a. Instantaneous turbulence structures}

An example of instantaneous streamwise velocity on a horizontal plane is shown at different heights in Fig. 12. Away from the surface, the turbulence shows the typical streak patterns of shear turbulence (Fig. 12c). These streaks are generated by the quasi-streamwise vortices (including the cane and hairpin vortices) in the log layer (Fig. 13). They are the main turbulence structures of the log layer over flat walls (e.g., del Álamo et al. 

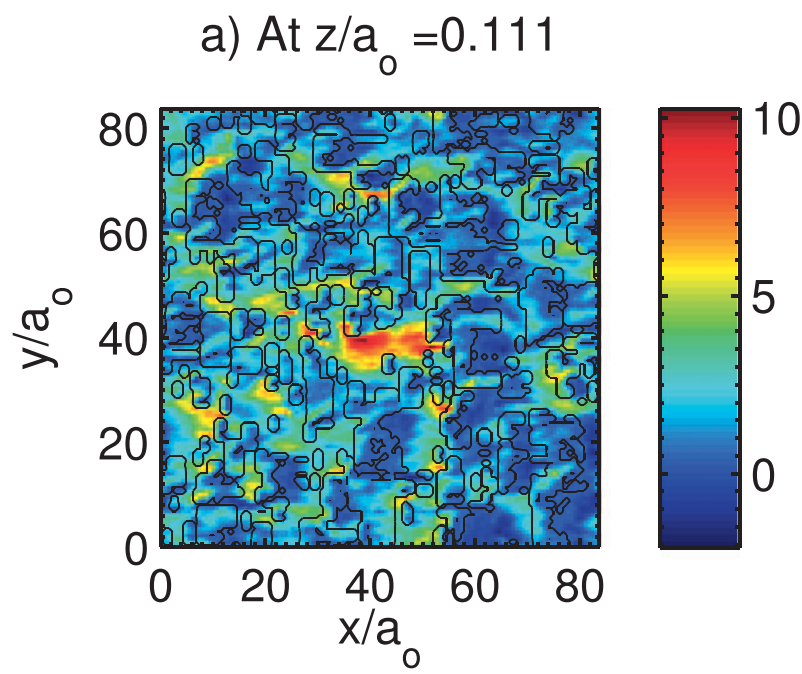

b) At $z / a_{0}=0.778$
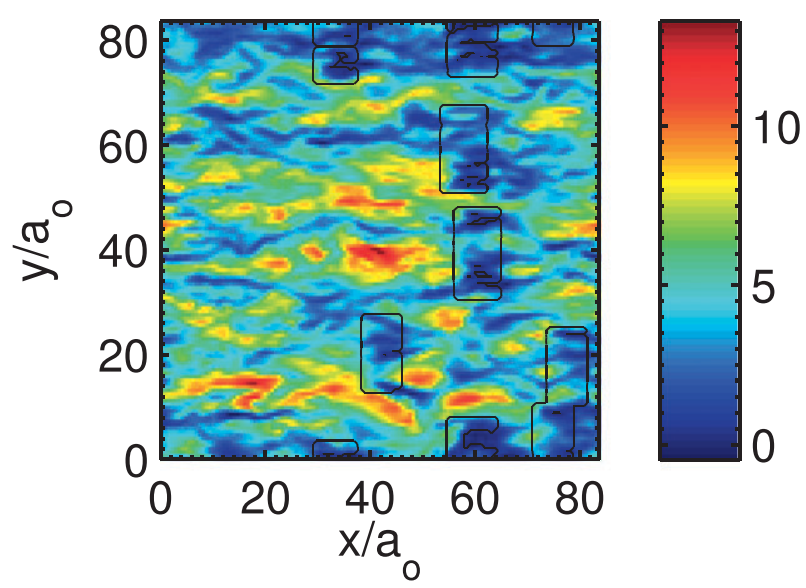

c) At $z / a_{0}=3.000$

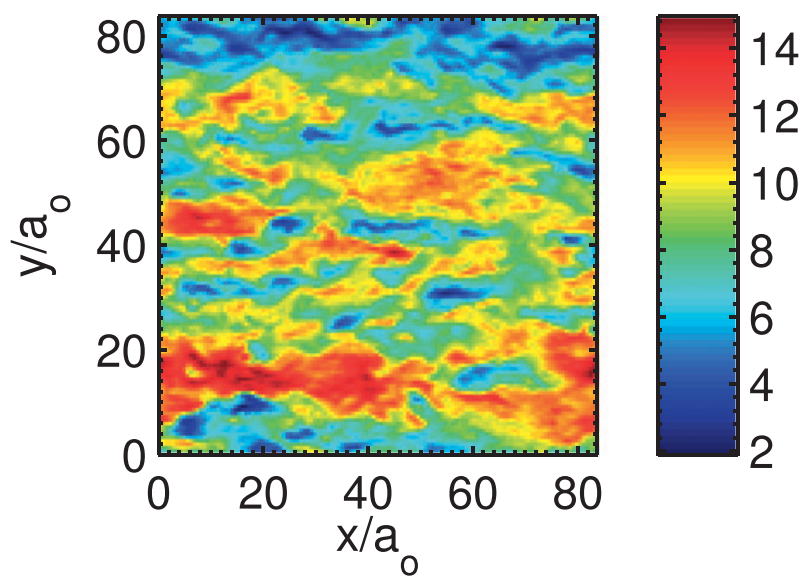

FIG. 12. Instantaneous snapshots of $u / U_{*}$ at different heights. The black contours show areas where the breaker forcing appears. The case shown is with wave age $=0.5, U_{*}=2 \mathrm{~m} \mathrm{~s}^{-1}, C_{d}^{\mathrm{BR}}=1.0$, and the baseline (BAS) $\Lambda$.

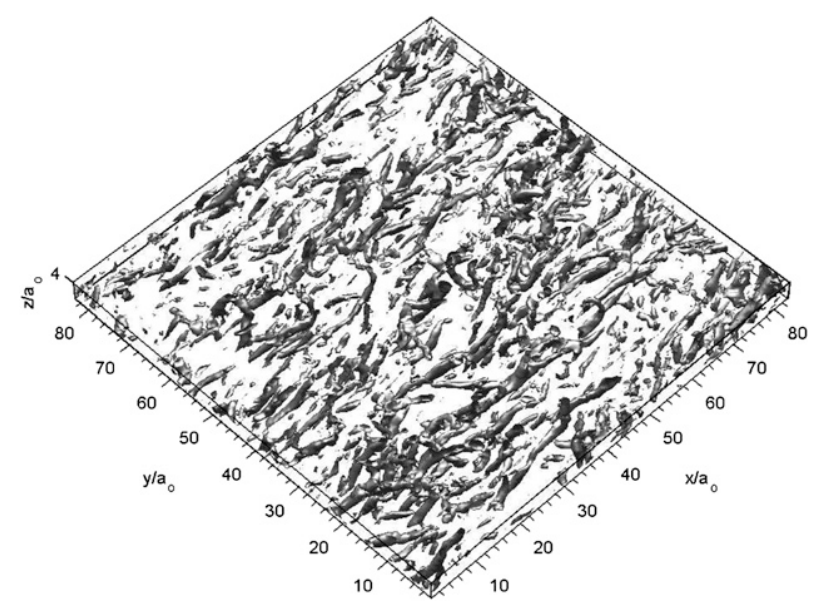

FIG. 13. Vortex cores below $z / a_{o}=4$. The vortex cores are identified using the scheme proposed by Chakraborty et al. (2005). The case shown is with wave age $=0.5, U_{*}=2 \mathrm{~m} \mathrm{~s}^{-1}, C_{d}^{\mathrm{BR}}=1.0$, and the baseline (BAS) $\Lambda$.

2006; Tomkins and Adrian 2005) and rough walls (e.g., Lee et al. 2009; Volino et al. 2007).

In contrast, in Figs. 12a,b the turbulence near the surface is strongly modified by the breaker-induced wakes, and the typical streak patterns no longer exist. The wakes can be identified by the low or negative winds in and past the areas where breaker forcing appears. The wakes show strong three dimensionality (along-crest variability) and are very transient. These features are consistent with the PIV observations of breaker-induced wakes (Reul et al. 2008). Among the wakes, there are sporadic regions of very high wind. These gusty regions roughly match the gusty regions at higher elevations unless the flow separation bubbles prevent such gusty motions near the surface. This suggests that a gust in the WBL comes from outside the WBL in the form of a sweep (i.e., a motion with $\bar{u}^{\prime}>0$ and $\bar{w}^{\prime}<0$ ) made by the large-scale quasi-streamwise vortices.

The mixing-layer-type turbulence structures, often seen in canopy-layer flows (Finnigan 2000), are not observed in our results (Fig. 13) even though there is a weak inflection of the mean wind profile very close to the surface (Fig. 7b). The absence of such structures with a mean wind shear inflection is also reported in a DNS study of a flow over transverse $k$-type roughness (Ikeda and Durbin 2007).

\section{b. TKE and variances}

In the following, we will investigate how the breaker density affects the turbulence characteristics. Figures 14 and 15 show snapshots of some key turbulent quantities for the dense case and the sparse case at wave age 0.5. Included are streamwise turbulent wind $\bar{u}^{\prime} / U_{*}$, net TKE 

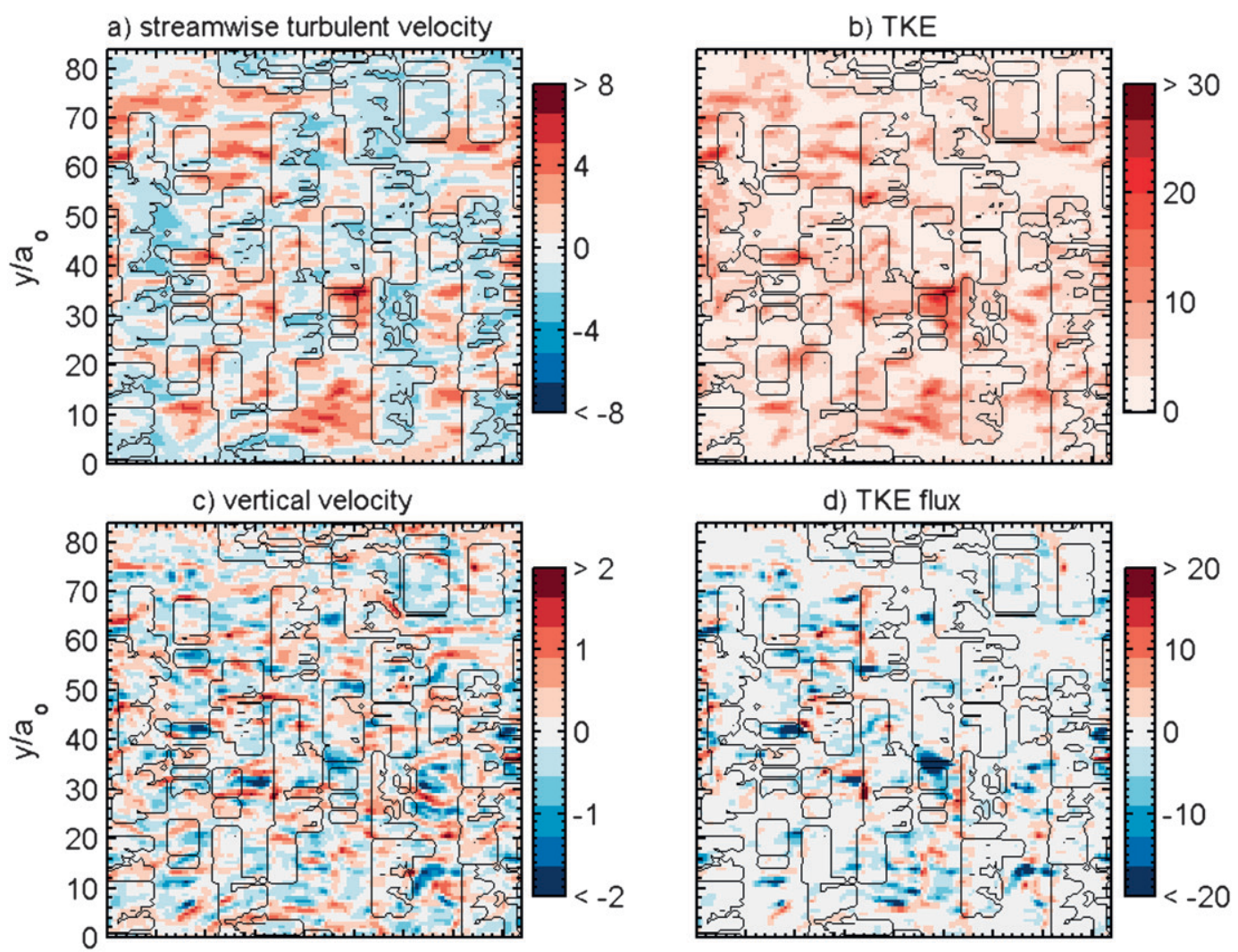

e) ejection(red) \& sweep(blue)
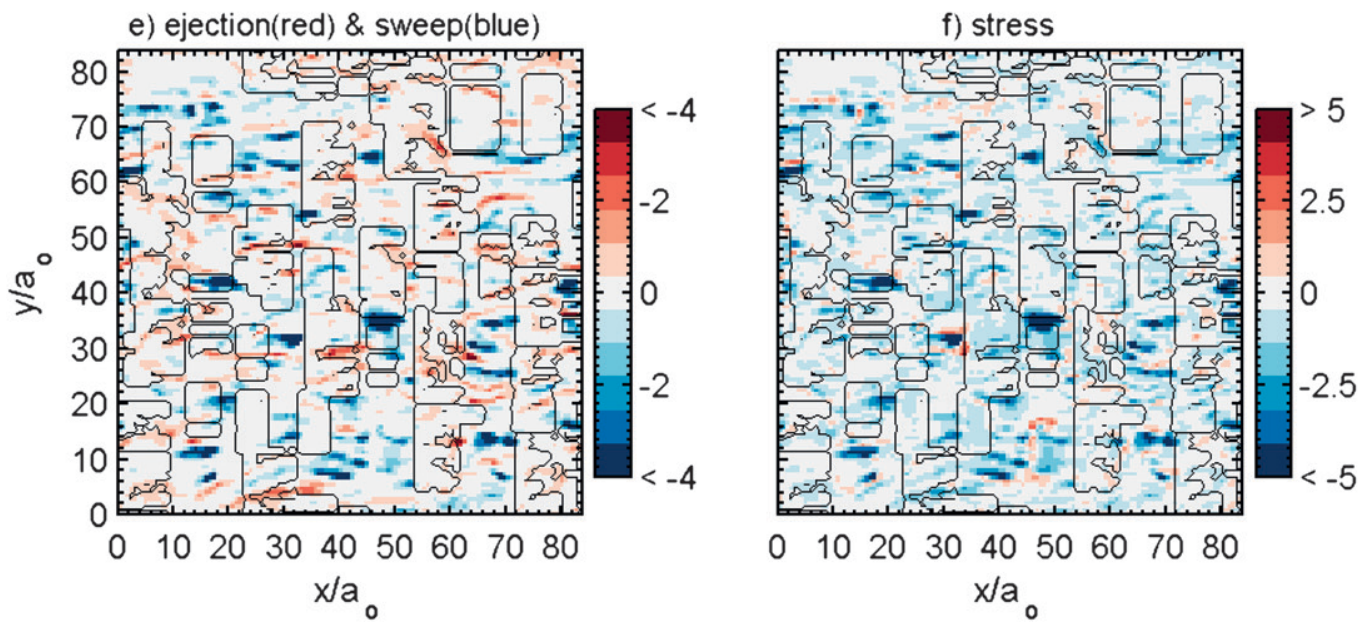

FIG. 14. Instantaneous turbulence fields on a horizontal plane near the middle of the WBL $\left(z / a_{o}=5 / 9\right)$ with wave age $=0.5, U_{*}=2 \mathrm{~m} \mathrm{~s}^{-1}, C_{d}^{\mathrm{BR}}=1.0$, and the dense (DEN) $\Lambda$ : (a) $u^{\prime} / U_{*} ;$ (b) $\left(E_{\mathrm{RT}}+e\right) / U_{*}^{2} ;$ (c) $w^{\prime} / U_{*} ;$ (d) $f_{T} / U_{*}^{3}$, defined in Eq. (11); (e) sweep $\left(u^{\prime} w^{\prime} / U_{*}^{2}\right.$, where $u^{\prime}>0$ and $\left.w^{\prime}<0\right)$ and ejection $\left(u^{\prime} w^{\prime} / U_{*}^{2}\right.$, where $u^{\prime}<0$ and $\left.w^{\prime}>0\right)$; and (f) $\left(\bar{u}^{\prime} \bar{w}^{\prime}+\bar{R}_{13}\right) / U_{*}^{2}$.

$\left(E_{\mathrm{RT}}+\bar{e}\right) / U_{*}^{2}$, vertical velocity $\bar{w}^{\prime} / U_{*}$, TKE flux $f_{T} / U_{*}^{3}$, ejections (i.e., motions with $\bar{u}^{\prime}<0$ and $\bar{w}^{\prime}>0$ ) and sweeps (i.e., motions with $\bar{u}^{\prime}>0$ and $\bar{w}^{\prime}<0$ ) expressed as $\bar{u}^{\prime} \bar{w}^{\prime} / U_{*}^{2}$, and net stress $\left(\bar{u}^{\prime} \bar{w}^{\prime}+\tau_{13}\right) / U_{*}^{2}$.

The TKE behaves quite differently between the dense and sparse cases. In the dense case, Fig. 14 shows a high correlation among the sporadic gusts (red spots in 14a), large TKE (red spots in 14b), downward TKE flux (blue spots in 14d), and sweeps (blue spots in 14e). This shows that the TKE inside the wave boundary layer is mostly due to the sporadic gusts, and this gust TKE is carried down into the WBL from outside by the sweeping motion associated with the large-scale quasi-streamwise vortices. The TKE of the wakes is much less than the gust 
a) streamwise turbulent velocity

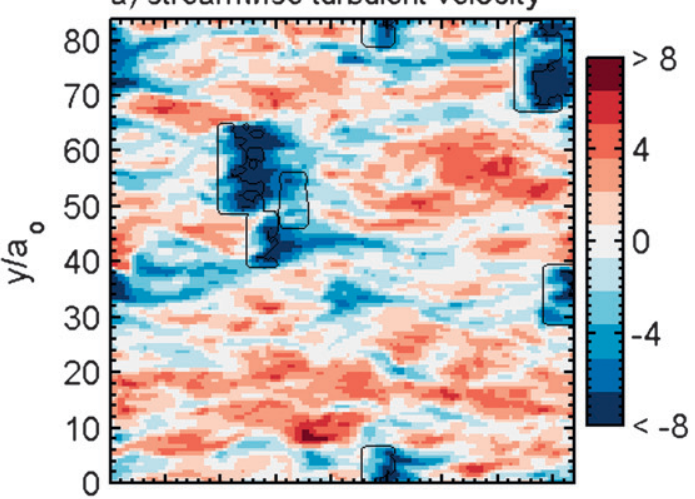

c) vertical velocity

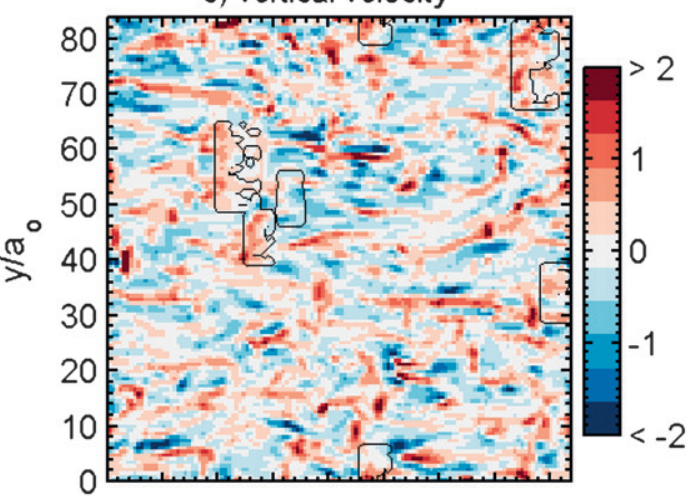

e) ejection(red) \& sweep(blue)

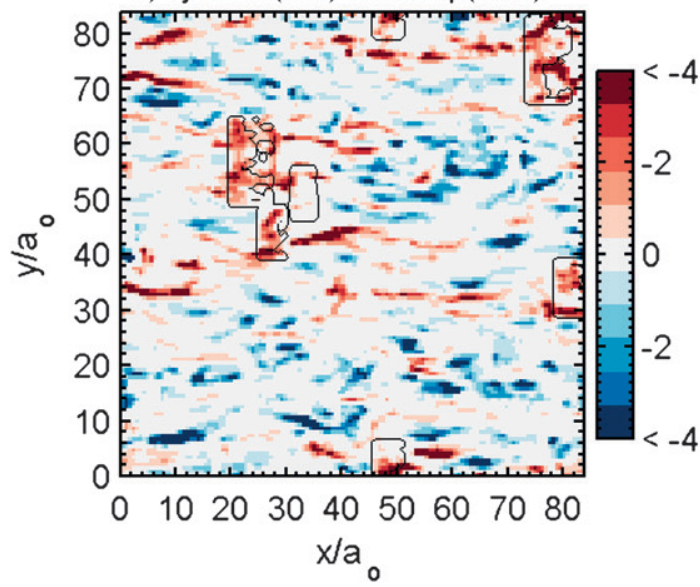

b) TKE

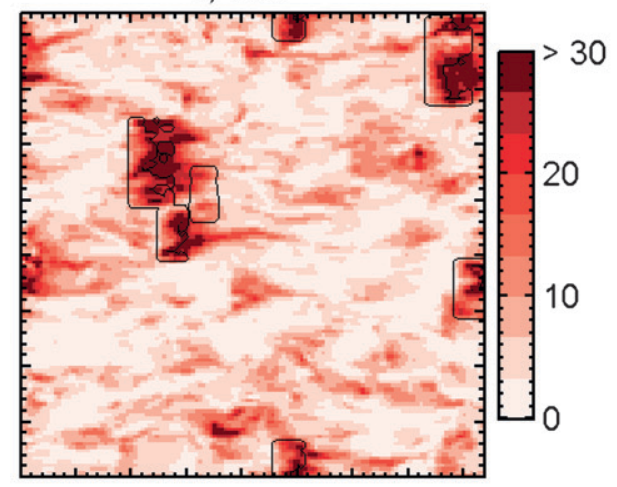

d) TKE flux

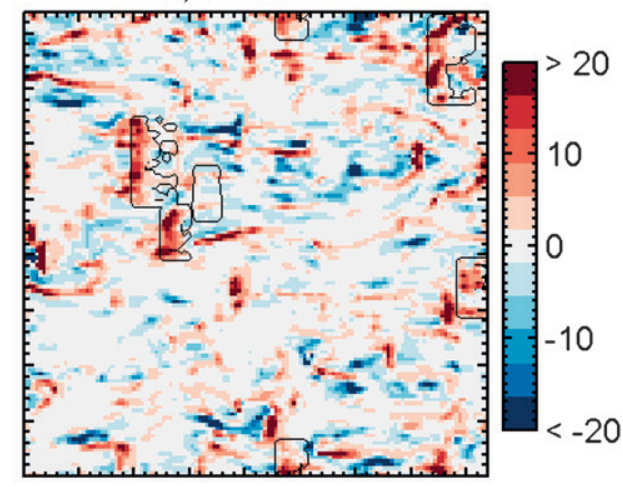

\section{f) stress}

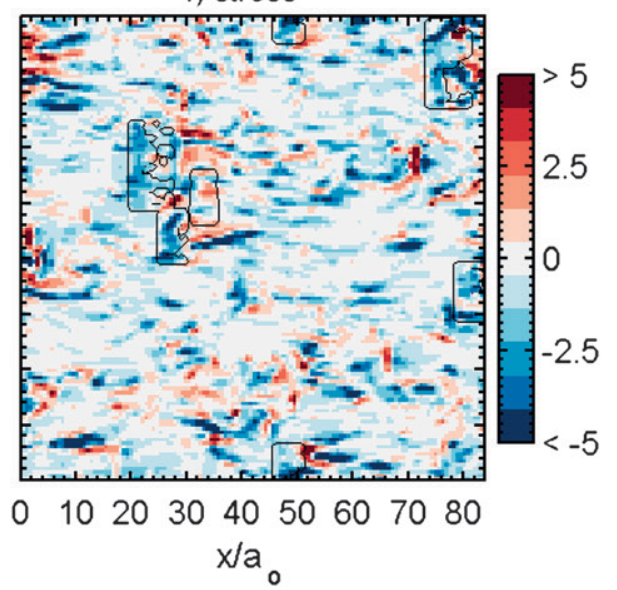

FIG. 15. As in Fig. 14 but with the sparse (SPA) $\Lambda$.

TKE because the wakes cover a large part of the WBL and the mean (horizontally averaged) wind speed is close to the wind speed in the wakes. Hence, the deviations $\left|\bar{u}^{\prime}\right|$ in the wakes are small (Fig. 14a), and the TKE is small as well.

In the sparse case, in contrast, the deviations $\left|\bar{u}^{\prime}\right|$ from the mean wind are large inside the wakes because the mean wind is relatively large (Fig. 15a). Hence the wake turbulence carries more TKE than the sweeps (gusts) (Fig. 15b).
The increased dominance of the wake turbulence in the SPA case is also evident in the variances shown in Fig. 16. The breakers in the sparse WBL result in a very large $\left\langle\bar{u}^{\prime} \bar{u}^{\prime}\right\rangle$ whereas breakers in the dense WBL make the flow more uniform with a much smaller $\left\langle\bar{u}^{\prime} \bar{u}^{\prime}\right\rangle$. The variance of the cross-stream velocity $\left\langle\bar{v}^{\prime} \bar{v}^{\prime}\right\rangle$ stays relatively high inside the WBL in all cases because the breaker form drag is anisotropic as explained earlier. 


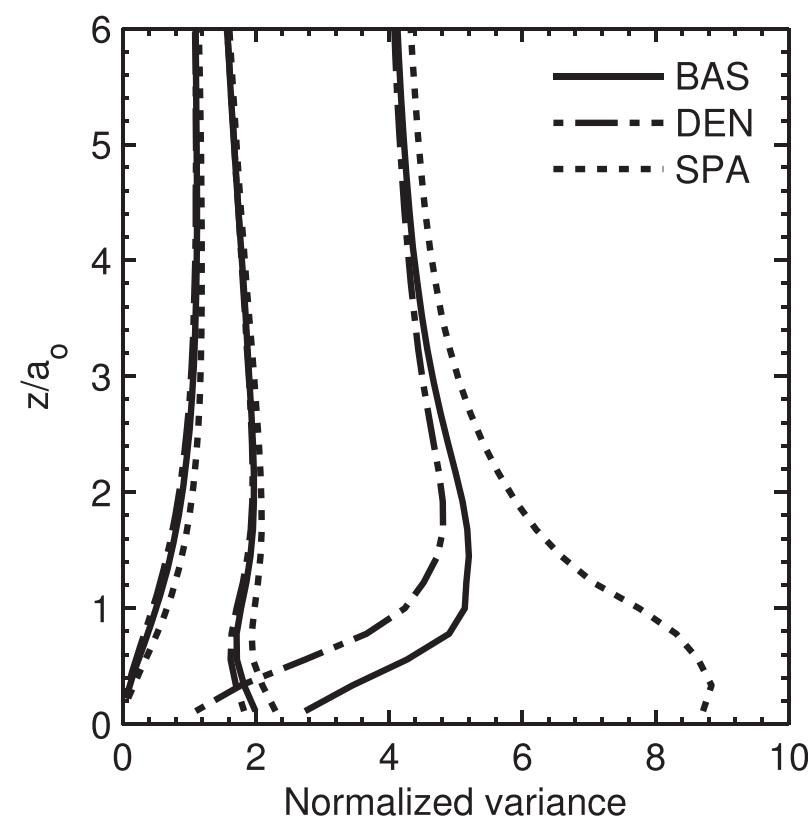

FIG. 16. Normalized variances $\left\langle\bar{u}^{\prime} \bar{u}^{\prime}\right\rangle / U_{*}^{2}$ (largest), $\left\langle\bar{v}^{\prime} \bar{v}^{\prime}\right\rangle / U_{*}^{2}$ (intermediate), $\left\langle\bar{w}^{\prime} \bar{w}^{\prime}\right\rangle / U_{*}^{2}$ (smallest). Cases shown are with wave age $=$ $0.5, U_{*}=2 \mathrm{~m} \mathrm{~s}^{-1}, C_{d}^{\mathrm{BR}}=1.0$, and three different $\Lambda$ (DEN, BAS, and SPA).

\section{c. Wake production: Energy conversion due to breaker form drag}

As explained in section $2 \mathrm{a}$, the terms representing work done by the breaker form drag satisfy the following conservation equation:

$$
\langle\bar{u}\rangle\left\langle\sum_{m} \bar{A}_{1}^{m}\right\rangle-\left\langle\sum_{m} c_{i} \bar{A}_{i}^{m}\right\rangle+\left\langle P_{\mathrm{W}}^{\mathrm{R}}\right\rangle+\left\langle P_{\mathrm{W}}^{\mathrm{SGS}}\right\rangle=0 .
$$

The first term represents the rate of energy loss in the mean flow energy $\left\langle E_{\mathrm{M}}\right\rangle$ by action of the drag. Since $\bar{A}_{1}^{m} \leq 0$ everywhere, the first term is always negative. The second term is the energy transfer to the breakers via the work done by the form drag and is always positive (i.e., waves gain energy). The third term $\left\langle P_{\mathrm{W}}^{\mathrm{R}}\right\rangle=$ $\left\langle\sum_{m} \bar{u}_{i}^{\prime} \bar{A}_{i}^{m}\right\rangle$ is the rate of work done on the resolved-scale turbulence by the form drag and can be positive or negative. For example, resolved-scale gusts have $\bar{u}^{\prime}>0$, and the breakers do work against them $\left(\bar{u}^{\prime} \bar{A}_{1}^{m} \leq 0\right)$. Hence, the gusts lose energy, and that energy is transferred to the breakers and the SGS wake turbulence. In contrast, $\bar{u}^{\prime}$ is negative inside a wake (Figs. 14a and 15a). Thus, the resolved wake turbulence gains energy $\left(\bar{u}^{\prime} \bar{A}_{1}^{m} \geq 0\right)$ from the mean flow. The term $\left\langle P_{\mathrm{W}}^{\mathrm{R}}\right\rangle$ is the average of these processes and is positive when the energy gain in the resolved wake turbulence is more than the energy loss in the resolved gusts, and vice versa.

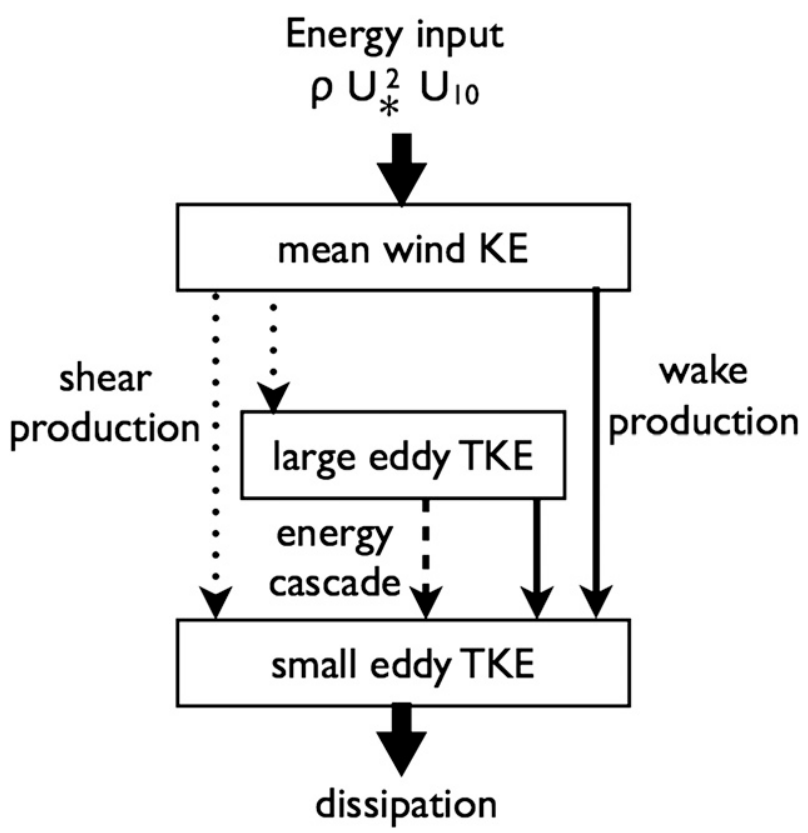

FIG. 17. Schematic showing main pathways for energy transfer.

Lastly, the fourth term of Eq. (15) is the SGS wake production term and is always zero or positive, as discussed in section 2a. In summary, Eq. (15) states that, when large-scale energetic motions (viz., the mean flow and gusts) hit breakers, they lose energy. Part of that lost energy is transferred to the breakers and the rest is converted to resolved-scale and SGS wake turbulence. Because the size of the wake turbulence roughly scales with the breaker height, the wake turbulence induced by short breakers is close to the viscous dissipation scale. This direct conversion of the mean flow energy and the large-scale TKE to the dissipative-scale TKE shortcuts the usual energy cascade and leads to large energy dissipation (Fig. 17). Such an effect of roughness elements has been well recognized in studies of canopy layers (Finnigan 2000). It is a critical mechanism for rough surfaces to dissipate large amounts of energy.

Figure 18 shows the energy conversion, Eq. (15), for the three cases of DEN, BAS, and SPA. There are significant differences in the wake production and the mean wind energy loss near the surface. In the dense breaker case (Fig. 18a), the rate of the mean-flow energy loss decreases near the surface because the mean flow is very small near the surface (Fig. 7). In contrast, when the breakers are sparse, the mean flow very near the surface is about five times larger (Fig. 7). As a result, both longer and shorter breakers are well exposed to high wind and contribute greatly to the conversion from the large-scale motions to the wake turbulence. 

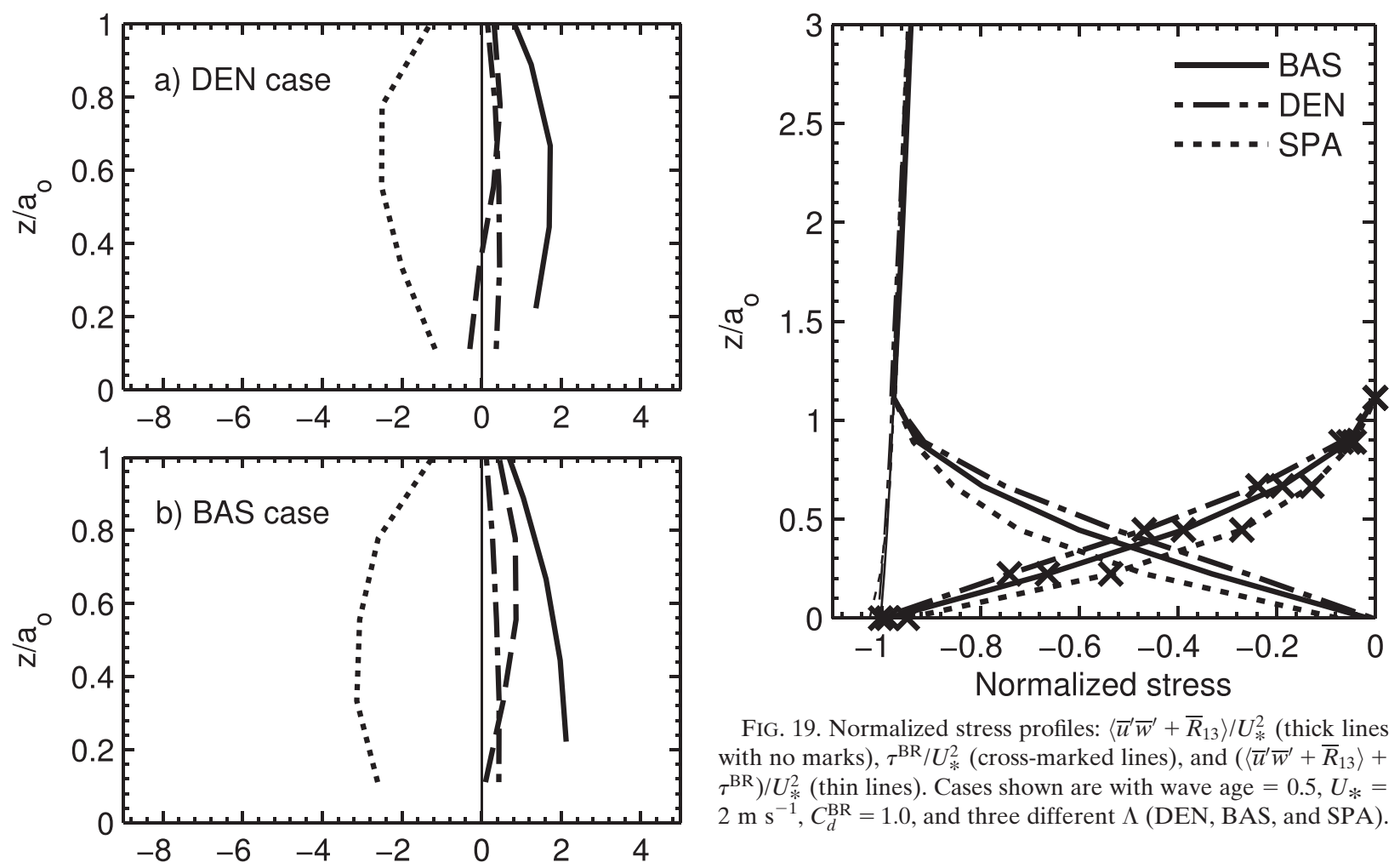

FIG. 19. Normalized stress profiles: $\left\langle\bar{u}^{\prime} \bar{w}^{\prime}+\bar{R}_{13}\right\rangle / U_{*}^{2}$ (thick lines with no marks), $\tau^{\mathrm{BR}} / U_{*}^{2}$ (cross-marked lines), and $\left(\left\langle\bar{u}^{\prime} \bar{w}^{\prime}+\bar{R}_{13}\right\rangle+\right.$ $\left.\tau^{\mathrm{BR}}\right) / U_{*}^{2}$ (thin lines). Cases shown are with wave age $=0.5, U_{*}=$ $2 \mathrm{~m} \mathrm{~s}^{-1}, C_{d}^{\mathrm{BR}}=1.0$, and three different $\Lambda$ (DEN, BAS, and SPA $)$

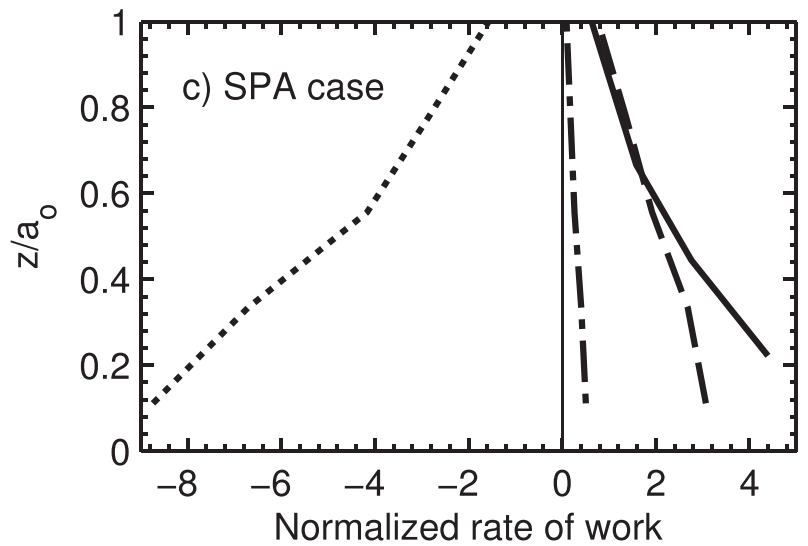

FIG. 18. Conversion of energy due to the breaker forcing, four terms of Eq. (15): $\langle\bar{u}\rangle\left\langle\sum_{m} \bar{A}_{1}^{m}\right\rangle$ (dot), $\left\langle-\sum_{m} c_{i} \bar{A}_{i}^{m}\right\rangle$ (dash-dot), $\left\langle P_{\mathrm{W}}^{\mathrm{R}}\right\rangle$ (dash), and $\left\langle P_{\mathrm{W}}^{\mathrm{SGS}}\right\rangle$ (solid). All terms are normalized by $U_{*}^{3} / a_{o}$ and are zero above $z / a_{o}=1$ as no drag appears there. Cases shown are with wave age $=0.5, U_{*}=2 \mathrm{~m} \mathrm{~s}^{-1}, C_{d}^{\mathrm{BR}}=1.0$, and three different $\Lambda$ (DEN, BAS, and SPA).

\section{d. Shear production and stress}

As shown in Fig. 10, the shear production $P_{\mathrm{MS}}^{\mathrm{R}}+$ $P_{\mathrm{MS}}^{\mathrm{SGS}}=-\left\langle\bar{u}^{\prime} \bar{w}^{\prime}+\bar{R}_{13}\right\rangle\langle\bar{u}\rangle / \partial z$ decreases significantly inside the WBL in all cases. This is because the force exerted by breakers impedes both wind shear $\partial\langle\bar{u}\rangle / \partial z$ (Fig. 5 and 7) and Reynolds shear stress $\left\langle\bar{u}^{\prime} \bar{w}^{\prime}+\bar{R}_{13}\right\rangle$ well inside the WBL. The reduction of the Reynolds shear stress is an inevitable result of the momentum budget

$$
\frac{d\left\langle\bar{u}^{\prime} \bar{w}^{\prime}+\bar{R}_{13}\right\rangle}{d z}=-\frac{\partial \bar{P}}{\partial x}+\left\langle\sum_{m} \bar{A}_{1}^{m}\right\rangle
$$

obtained by horizontally averaging the momentum equation (1) in a statistically steady state. Above $z / a_{o}=$ 1 , the breaker forcing $\left\langle\sum_{m} \bar{A}_{1}^{m}\right\rangle$ is zero, and the Reynolds stress profile is determined solely by the constant background mean pressure gradient forcing. Below $z / a_{o}=1,\left|\left\langle\sum_{m} \bar{A}_{1}^{m}\right\rangle\right|$ is much larger than $|-\partial \bar{P} / \partial x|$. Thus, the Reynolds stress inside the WBL is determined by the breaker forcing. As $\left\langle\sum_{m} \bar{A}_{1}^{m}\right\rangle$ is negative, the Reynolds stress is reduced toward the surface. The breaker forcing is often expressed in terms of the breaker stress $\tau^{\mathrm{BR}}$, where $d \tau^{\mathrm{BR}} / d z=-\left\langle\sum_{m} \bar{A}_{1}^{m}\right\rangle$. The breaker stress $\tau^{\mathrm{BR}}(z)$ represents the average air-sea momentum flux supported by the breaker forcing appearing above $z$. In terms of the breaker stress, Eq. (16) can be rewritten as

$$
\frac{d\left\langle\bar{u}^{\prime} \bar{w}^{\prime}+\bar{R}_{13}\right\rangle+\tau^{\mathrm{BR}}}{d z}=-\frac{\partial \bar{P}}{\partial x} .
$$

Examples of these stresses are shown in Fig. 19. In all cases $\tau^{\mathrm{BR}} / U_{*}^{2}$ reaches nearly -1 near the surface; that is, almost all air-sea momentum flux is supported by the breakers in our LES (i.e., more than $95 \%$ for most cases and about $90 \%$ for the SPA case).

The mechanisms of the turbulent momentum transfer are different depending on the breaker density. In the 


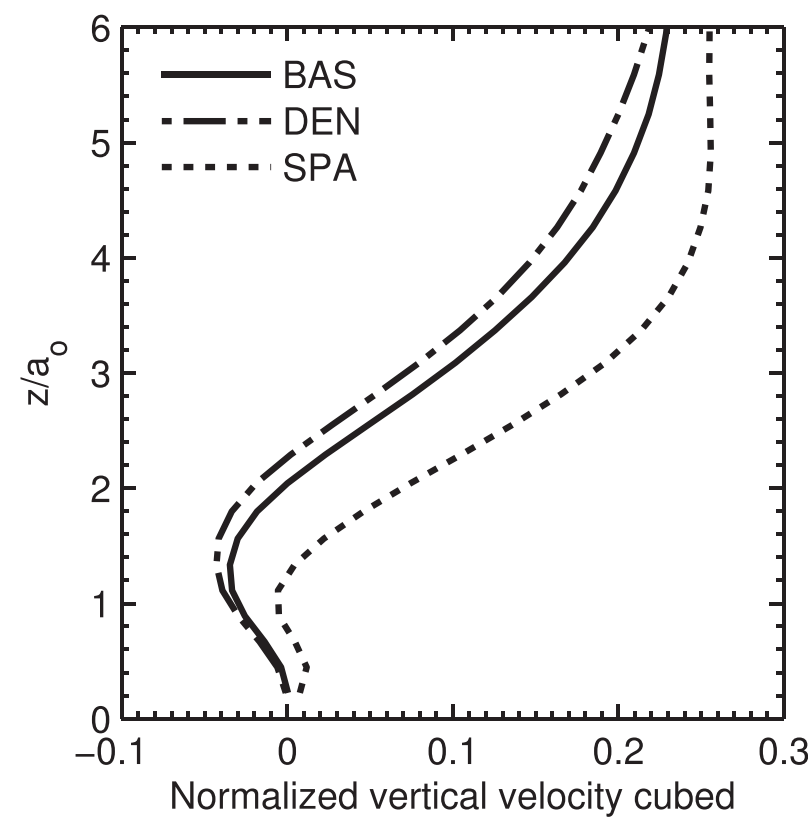

FIG. 20. Normalized vertical velocity cubed $\left\langle\bar{w}^{\prime} \bar{w}^{\prime} \bar{w}^{\prime}\right\rangle / U_{*}^{3}$ : cases shown are with wave age $=0.5, U_{*}=2 \mathrm{~m} \mathrm{~s}^{-1}, C_{d}^{\mathrm{BR}}=1.0$, and three different $\Lambda$ (DEN, BAS, and SPA).

dense breaker case, the downward momentum flux $\bar{u}^{\prime} \bar{w}^{\prime}+\bar{R}_{13}$ (the blue color in Fig. 14f) is not correlated with the wake turbulence (the blue color in Fig. 14a) but is mainly due to sweeps and ejections (the blue color and the red color in Figs. 14e) caused by the quasistreamwise vortices above the WBL. The sweeps (blue) are more vigorous than the ejections (red). Figure 20 also shows that the sweeps are more vigorous than the ejections, particularly in the upper part of the WBL. Here, when ejections $\left(\bar{w}^{\prime}>0\right)$ are stronger than sweeps $\left(\bar{w}^{\prime}<0\right),\left\langle\bar{w}^{\prime 3}\right\rangle / U_{*}^{3}$ becomes more positive, and vice versa. This is opposite to the state in the logarithmic layer above, where ejections are larger than sweeps.

In the sparse breaker case, inside a breaker-induced separation the spanwise vortex (Fig. 2) causes a large ejection $\left(\bar{u}^{\prime}<0, \bar{w}^{\prime}>0\right)$ where the swirling motion goes up (the red color inside the breaker forcing regions in Fig. 15e), yielding a large downward momentum flux (the blue color inside the breaker forcing regions in Fig. 15f). However, near the reattachment point of the same vortex, the vertical velocity changes its sign $\left(\bar{u}^{\prime}<0, \bar{w}^{\prime}<0\right)$ and yields upward momentum flux (the red color appearing right behind the form drag regions in Fig. 15f). On average, these negative and positive momentum fluxes in the wakes cancel out. Therefore, the wakes contribute little to the Reynolds shear stress in the sparse case as well. Outside the wakes, the ejections and sweeps are more regular (Fig. 20) compared to the dense breaker case, reflecting less disruption of the regular quasi-streamwise vortex processes (Figs. 15e and 15f).

\section{e. Summary}

The effects of breakers on the WBL turbulence characteristics are summarized as follows.

(i) There are two major eddy types in the WBL: namely, quasi-streamwise vortices (regular shear turbulence) and wake turbulence (due to breakers). The statistical properties of the near-surface turbulence result from a mixture of these two eddies.

(ii) Breakers modify the near-surface turbulence by 1) preventing quasi-streamwise vortex motions in the WBL and 2) generating wake turbulence.

(iii) Breaker-induced flow separation bubbles shelter smaller-scale breakers.

Therefore, the density of breakers significantly alters the detailed turbulence characteristics. In the sparse case, the breakers are well exposed to high wind and generate strong wake turbulence. In the dense case, the breakers are not exposed to high wind because a large part of the WBL is covered with wakes. The wake turbulence from each breaker is weaker and does not contribute as much to the overall TKE. Instead, the turbulence characteristics are more associated with the quasi-streamwise vortices.

\section{Concluding remarks}

Using LES, which resolves individual wakes generated by breaking waves, the impacts of breaker form drag on airflow turbulence and drag coefficient have been studied at young sea states in hurricane-strength winds. Overall, the simulated $C_{D 10}$ falls in the range between 0.002 and 0.003 . It remains nearly constant at high winds if the breaker distribution is kept roughly the same as the wind speed increases. The relatively low $C_{D 10}$ results because the normalized TKE dissipation rate integrated over the atmospheric wave boundary layer (WBL) is relatively large in high winds. The main impact of the breaker form drag on the TKE budget is to impede the shear production and, instead, produce small-scale wake turbulence by converting the kinetic energy of the mean wind and large-scale gusts. This shortcut of the usual energy cascade has been known in canopy-layer studies but has been overlooked in previous WBL studies. Because the increased wake production replaces the decreased shear production, the net TKE production stays relatively large. This results in the large dissipation in the WBL at high winds. The LES results show that at hurricane-strength winds more than $90 \%$ of the air-sea momentum flux is due to the form 
drag of the breakers; that is, the contributions from the surface viscous stress and the nonbreaking wave form drag are small. Our results also suggest that common parameterizations for the mean wind profile and the TKE dissipation used in previous Reynolds-averaged Navier-Stokes WBL models may not be valid.

When the breaker density is high, a large fraction of the WBL is covered with wakes, and the mean wind speed approaches the wind speed inside the wakes. Since breakers are effectively sheltered by other breakers, the wake turbulence is relatively weak. In contrast, when the breaker density is low, the difference between the mean wind and the wind speed inside the wakes becomes large, and the wake turbulence is stronger and becomes significant in the overall WBL turbulence characteristics. Since the sheltering effect can significantly alter the TKE budget, it should be explicitly accounted for in the RANS WBL framework as well.

In open ocean conditions the sea is more developed even at hurricane-strength winds (wave age is typically between 5 and 10, see Moon et al. 2004) and the results of this study are not directly applicable. At larger wave ages the breaking events of the dominant scale waves are likely reduced and the contribution of the form drag from nonbreaking waves becomes increasingly important (Kukulka and Hara 2008b). It is therefore of great interest to investigate to what extent the wake turbulence generation mechanism by breaking waves remains significant in the overall TKE budget over more developed seas. This will be the subject of our next study.

Acknowledgments. This work was supported by the U.S. National Science Foundation (Grant OCE0824906). We used the computational resources at National Center for Atmospheric Research (NCAR).

\section{APPENDIX}

\section{Upper and Lower Bounds of Breaking Distribution}

We estimate reasonable upper and lower bounds of $\Lambda(k)$ (integrated in all angles) based on its relationship with the wave saturation spectrum $B(k)=k^{3} \phi(k)$, where $\phi(k)$ is the wavenumber spectrum (integrated in all angles). If we represent a wave field with a finite number of sinusoidal wave trains of different discrete wavenumbers, then for each wave train $\phi(k) \Delta k=a^{2} / 2$ where $a$ is the amplitude of the wave train at $k$. Thus,

$$
\frac{\Delta k}{k}=\frac{a^{2}}{2 k \phi(k)}=\frac{(a k)^{2}}{2 B(k)} \text {. }
$$

On the other hand, the length of breaking crests per unit horizontal area $\Lambda(k) \Delta k$ of the same wave train should not exceed the total (breaking and nonbreaking) crest length per unit horizontal area, which equals $\lambda / \lambda^{2}$, where $\lambda$ is the wavelength. Thus, the upper limit of $\Lambda(k)$ (i.e., when $100 \%$ of waves break) may be estimated by $\Lambda(k) \Delta k<1 / \lambda$ or equivalently

$$
\Lambda(k)<\frac{k}{2 \pi \Delta k}=\frac{B(k)}{\pi(a k)^{2}} .
$$

If we assume that most waves are breaking and the wave slope $a k$ is close to the critical wave slope 0.3 , which is the typical wave slope of breakers, we obtain

$$
\Lambda(k)=\frac{B(k)}{0.09 \pi} .
$$

In open ocean conditions under moderate winds, $B(k)$ is $0.008 \pm 0.002$ (Romero and Melville 2010) for short gravity waves. In wind wave tanks $B(k)$ can be as large as 0.1 near the spectral peak (e.g., Caulliez et al. 2008; Jessup and Phadnis 2005). We therefore set the upper and lower bounds of $B$ as 0.1 and 0.006 , and the corresponding upper and lower bounds of $\Lambda$ as 0.35 and 0.021 , as shown in Fig. 3. The figure also shows the laboratory experimental data of microwave breaking with the wind speed of $9.6 \mathrm{~m} \mathrm{~s}^{-1}$ and peak wavelength of $0.156 \mathrm{~m}$ (Jessup and Phadnis 2005). (The uncertainty in their data is due to the uncertainty in the conversion between the measured $c$ and $k$ and the uncertainty in the wave age.) Note that the $\Lambda$ values at wind speeds $40-53 \mathrm{~m} \mathrm{~s}^{-1}$ (i.e., conditions of this study) are likely higher than the observed values of Jessup and Phadnis (2005) with much lower wind speeds.

\section{REFERENCES}

Babanin, A. V., and Y. P. Soloviev, 1998: Field investigation of transformation of the wind wave frequency spectrum with fetch and the stage of development. J. Phys. Oceanogr., 28, 563-576.

Bao, J.-W., C. W. Fairall, S. A. Michelson, and L. Bianco, 2011: Parameterizations of sea-spray impact on the airsea momentum and heat fluxes. Mon. Wea. Rev., 139, 3781-3797.

Barenblatt, G. I., A. J. Chorin, and V. M. Prostokishin, 2005: A note concerning the lighthill sandwich model of tropical cyclones. Proc. Natl. Acad. Sci. USA, 102, 11 148-11 150.

Bell, M. M., M. T. Montgomery, and K. A. Emanuel, 2012: Air-sea enthalpy and momentum exchange at major hurricane wind speeds observed during CBLAST. J. Atmos. Sci., 69, 31973222.

Bianco, L., J.-W. Bao, C. W. Fairall, and S. A. Michelson, 2011: Impact of sea-spray on the atmospheric surface layer. Bound.Layer Meteor., 140, 361-381.

Britter, R. E., and S. R. Hanna, 2003: Flow and dispersion in urban areas. Annu. Rev. Fluid Mech., 35, 469-496.

Caulliez, G., V. Makin, and V. Kudryavtsev, 2008: Drag of the water surface at very short fetches: Observations and modeling. J. Phys. Oceanogr., 38, 2038-2055. 
Chakraborty, P., S. Balachandar, and R. J. Adrian, 2005: On the relationships between local vortex identification schemes. J. Fluid Mech., 535, 189-214.

Deardorff, J. W., 1980: Stratocumulus-capped mixed layers derived from a three-dimensional model. Bound.-Layer Meteor., 18, $495-527$.

del Álamo, J. C., J. Jiménez, P. Zandonade, and R. D. Moser, 2006: Self-similar vortex clusters in the turbulent logarithmic region. J. Fluid Mech., 561, 329-358.

Donelan, M. A., B. K. Haus, N. Reul, W. J. Plant, M. Stiassnie, H. C. Graber, O. B. Brown, and E. S. Saltzman, 2004: On the limiting aerodynamic roughness of the ocean in very strong winds. Geophys. Res. Lett., 31, L18306, doi:10.1029/ 2004GL019460.

Edson, J., and Coauthors, 2007: The coupled boundary layers and air-sea transfer experiment in low winds. Bull. Amer. Meteor. Soc., 88, 341-356.

Finnigan, J., 2000: Turbulence in plant canopies. Annu. Rev. Fluid Mech., 32, 519-571.

French, J. R., W. M. Drennan, J. A. Zhang, and P. G. Black, 2007: Turbulent fluxes in the hurricane boundary layer. Part I: Momentum flux. J. Atmos. Sci., 64, 1089-1102.

Holthuijsen, L. H., M. D. Powell, and J. D. Pietrzak, 2012: Wind and waves in extreme hurricanes. J. Geophys. Res., 117, C09003, doi:10.1029/2012JC007983.

Ikeda, T., and P. A. Durbin, 2007: Direct simulations of a roughwall channel flow. J. Fluid Mech., 571, 235-263.

Jessup, A. T., and K. R. Phadnis, 2005: Measurement of the geometric and kinematic properties of microscale breaking waves from infrared imagery using a piv algorithm. Meas. Sci. Technol., 16, 1961-1969.

Kleiss, J. M., and W. K. Melville, 2011: The analysis of sea surface imagery for whitecap kinematics. J. Atmos. Oceanic Technol., 28, 219-243.

Kudryavtsev, V. N., and V. K. Makin, 2007: Aerodynamic roughness of the sea surface at high winds. Bound.-Layer Meteor., 125, 289-303.

— and - 2011: Impact of ocean spray on the dynamics of the marine atmospheric boundary layer. Bound.-Layer Meteor., 140, 383-410.

Kukulka, T., and T. Hara, 2008a: The effect of breaking waves on a coupled model of wind and ocean surface waves. Part I: Mature seas. J. Phys. Oceanogr., 38, 2145-2163.

$\longrightarrow$, and — 2008b: The effect of breaking waves on a coupled model of wind and ocean surface waves. Part II: Growing seas. J. Phys. Oceanogr., 38, 2164-2184.

,-- , and S. E. Belcher, 2007: A model of the air-sea momentum flux and breaking-wave distribution for strongly forced wind waves. J. Phys. Oceanogr., 37, 1811-1828.

Large, W. G., and S. Pond, 1981: Open ocean momentum flux measurements in moderate to strong winds. J. Phys. Oceanogr., 11, 324-336.
Lee, J. H., S.-H. Lee, K. Kim, and H. J. Sung, 2009: Structure of the turbulent boundary layer over a rod-roughened wall. Int. J. Heat Fluid Flow, 30, 1087-1098.

Makin, V. K., 2005: A note on the drag of the sea surface at hurricane winds. Bound.-Layer Meteor., 115, 169-176.

Moeng, C.-H., 1984: A large-eddy-simulation model for the study of planetary boundary-layer turbulence. J. Atmos. Sci., 41, 2052-2062.

Moon, I. J., T. Hara, and I. Ginis, 2004: Effect of surface waves on charnock coefficient under tropical cyclones. Geophys. Res. Lett., 31, L20302, doi:10.1029/2004GL020988.

Mueller, J. A., and F. Veron, 2009: Nonlinear formulation of the bulk surface stress over breaking waves: Feedback mechanisms from air-flow separation. Bound.-Layer Meteor., 130, 117-134.

Phillips, O., 1985: Spectral and statistical properties of the equilibrium range in wind-generated gravity waves. J. Fluid Mech., 156, 505-531.

Powell, M. D., P. J. Vickery, and T. A. Reinhold, 2003: Reduced drag coefficient for high wind speeds in tropical cyclones. Nature, 422, 279-283.

Raupach, M. R., and R. H. Shaw, 1982: Averaging procedures for flow within vegetation canopies. Bound.-Layer Meteor., 22, 79_ 90.

Reul, N., H. Branger, and J. P. Giovanangeli, 2008: Air flow structure over short-gravity breaking water waves. Bound.-Layer Meteor., 126, 477-505.

Romero, L., and W. K. Melville, 2010: Airborne observations of fetch-limited waves in the Gulf of Tehuantepec. J. Phys. Oceanogr., 40, 441-465.

Shaw, R. H., and U. Schumann, 1992: Large-eddy simulation of turbulent flow above and within a forest. Bound.-Layer Meteor., 61, 47-64.

Soloviev, A., and R. Lukas, 2010: Effects of bubbles and sea spray on air-sea exchange in hurricane conditions. Bound.-Layer Meteor., 136, 365-376.

Sullivan, P. P., J. C. McWilliams, and C.-H. Moeng, 1994: A subgridscale model for large-eddy simulation of planetary boundarylayer flows. Bound.-Layer Meteor., 71, 247-276.

,$- \ldots$, and W. K. Melville, 2007: Surface gravity wave effects in the oceanic boundary layer: Large-eddy simulation with vortex force and stochastic breakers. J. Fluid Mech., 593, 405-452.

Suzuki, N., T. Hara, and P. P. Sullivan, 2011: Turbulent airflow at young sea states with frequent wave breaking events: Largeeddy simulation. J. Atmos. Sci., 68, 1290-1305.

Tomkins, C. D., and R. J. Adrian, 2005: Energetic spanwise modes in the logarithmic layer of a turbulent boundary layer. J. Fluid Mech., 545, 141-162.

Volino, R. J., M. P. Schultz, and K. A. Flack, 2007: Turbulence structure in rough- and smooth-wall boundary layers. J. Fluid Mech., 592, 263-293. 